\title{
Des modèles théoriques pour étudier l'activité de l'expert en sport
}

\author{
Anne-Claire Macquet et Philippe Fleurance \\ Revue Science et Motricité, 2006, 2, 58
}

\begin{abstract}
Résumé
Cette article a consisté en une approche épistémologique de l'activité de l'expert en
\end{abstract} sport. Deux types d'approches ont été distinguées: le premier s'est appuyé sur le modèle cognitiviste classique (Chase \& Simon, 1973) et le second sur le paradigme de l'action située (Suchman, 1987). Le premier a étudié les compétences spécifiques des experts ; les situations standardisées utilisées réduisaient le domaine d'expertise pour mieux le contrôler. Cette approche est apparue controversée : elle n'a pas pris en compte le contexte qui évolue constamment en sport. Elle n'a pas réussi à prendre en compte l'activité dans les situations habituelles de travail. Cette controverse a été à l'origine d'un cheminement théorique vers l'action située, puis vers deux approches en ergonomie. La première s'est éloignée radicalement de la position cognitiviste, en contestant l'idée de représentation dans ses rapports avec l'action contextualisée. Elle considère que le couplage individu / situation est opérationnellement clos et que la cognition est énactée. Les décisions sont émergeantes (Theureau, 1992). La seconde a renvoyé au modèle de la suffisance cognitive dans la planification, l'exécution et le contrôle de l'action. Ce modèle envisage une gestion dynamique du couplage et une planification de l'action associée à des émergences programmées (Amalberti, 2001).

Ces modèles distinctifs ont permis une meilleure compréhension de ce qui oriente et contrôle l'action sportive en milieu naturel.

Mots clés : expert - contexte - sens - situation dynamique - émergence- planification.

Some theoretical models to study expert activity in sport

Abstract

This study presented an epistemological overview on expert activity. Two approaches have been distinguished: the first one refered to a classical cognitivist model (Chase \& Simon, 1973), whereas the second one refered to the situated action paradigm (Suchman, 1987). The former approach studied skills used by experts. This approach used experimental and standardised situations in order to reduce the domain of expertise to control it more efficiently. However, this approach was controversial : it did not take into consideration the context that constantly changes in sport. It could therefore not be representative of the activity in usual work situations. This controversy has lead to a theoretical evolution toward situated action paradigm, later was comprised of two different models in ergonomics. The first ergonomic model refused the concept of representation in relation with contextualised action. There is a coupling between the actor and the environment which is closed. Cognition is enacted. Decisions emerges from this coupling (Theureau, 1992). The second ergonomic approach was referred as the cognitive sufficiency model in planning, execution and control of action. It suggests a dynamic management of coupling and a programming of action associated with planned emergence (Amalberti, 2001).

These different models could provide a better understanding of what guide and control action in context of performance.

Key words : expert - context - signification - dynamic situation - emergence - planification 
Actuellement, la référence à l'expert est un «point d'achoppement » pour étudier l'activité de l'homme au travail. L'expert est apparu comme une source d'information pour connaître les éléments constitutifs des modes d'adaptation efficaces et singuliers. Les modèles disponibles dans la littérature ont reposé sur différents postulats et méthodologies. Globalement, deux types d'études ont été distingués: le premier a fait référence à une approche cognitive valorisant la conception de l'individu conçu comme un système de traitement de l'information. Le second type a valorisé l'étude de l'acteur dans le contexte de son travail quotidien et a renvoyé à l'approche de l'action située (Suchman, 1987). Bien que les modèles cognitivistes aient été discutés (Sanders, 1986 ; Varela, 1989a), ils ont donné lieu à des études qui ont fourni des indications sur les processus qui caractérisent l'expertise (Chase \& Simon, 1973 ; Abernethy \& Russel,1987). Leurs résultats ont permis de mettre à jour des compétences spécifiques aux experts, à travers une démarche souvent comparative (expert / novice), dans des situations standardisées. Les travaux les plus récents en sport, se sont orientés dans différents axes : la recherche d'amélioration de la prise d'information à partir d'un entraînement spécifique (Abernethy, Wood \& Park, 1999), l'étude des rapports entre la perception et certaines émotions telle l'anxiété (Williams \& Eliott, 1999), ou les relations entre les perceptions et les contraintes liées à la tâche (Williams, 2003).

L'article a consisté en une revue de littérature portant sur les modèles et les recherches empiriques liées à l'étude de l'expert et en particulier de l'expert sportif. Les travaux les plus anciens se sont appuyés sur des modèles d'expertise variés (échecs, médecine...). Ils ont influencé les recherches plus récentes sur le sport, qui leur ont emprunté leurs problématiques et leurs paradigmes expérimentaux. Les résultats ont souvent été consistants, entre les différents domaines d'expertise. Ces travaux sont apparus utiles pour comprendre l'orientation des études sur l'expertise en sport, pour déterminer les caractéristiques générales de l'expert et pour envisager le développement de certains processus.

Au cours de cette revue, nous avons tenté de montrer qu'actuellement, une posture ancrée sur le courant de l'action située a permis de développer un point de vue pertinent sur l'étude de l'action en cours, c'est-à-dire dans le contexte de la performance, et ceci à trois niveaux : préalablement à l'action, pendant l'action (en proposant une conception particulière des rapports entre l'individu agissant et son contexte de pratique), après l'action au plan de l'analyse de l'action passée. Le cours d'action ou le cours d'expérience de l'athlète est devenu un objet d'étude accessible, de l'expert sportif en contexte.

Nous avons étudié les modèles théoriques issus de la psychologie cognitive à partir d'une analyse des postulats, des hypothèses et de la nature des recherches menées. Une réflexion sur les limites de ces approches nous a amené à évoquer une controverse entre des modèles centrés sur la mise à jour de compétences spécifiques en situations standardisées et des modèles visant à étudier l'action en situation naturelle. Enfin, nous nous sommes focalisés sur les modèles issus de l'ergonomie de langue française, à partir de leurs hypothèses, de leurs postulats (de leurs présupposés) sur la cognition ainsi que sur la nature des recherches menées.

\section{Le paradigme cognitiviste computationnel : l’origine de la controverse}

\section{Les fondements théoriques}

Le courant cognitiviste a trouvé son origine dans la cybernétique. Il repose sur l'intuition selon laquelle l'intelligence humaine serait proche du fonctionnement d'un ordinateur. A travers le postulat central du cognitivisme, le cerveau travaille sur des représentations qui ont une réalité physique, symbolique et sémantique. La cognition se fonde sur des computations 
de représentations symboliques, à savoir des calculs sur des représentations. La computation est sémantique ou représentationnelle (sinon le traitement des symboles serait totalement aléatoire). On a considéré que l'homme pouvait se représenter le monde d'une façon particulière.

L’approche cognitive classique a proposé une explication mécaniste de la production de l'action par la cognition. L'input renvoie aux informations prélevées dans l'environnement par les systèmes sensoriels, puis traitées à un niveau centralisé du système global ; elles donnent des indications sur l'état de l'environnement. L'output est constitué par un ensemble d'instructions transmises au système moteur (Temprado \& Famose, 1993 ; Sanders, 1986). Dans cette conception du canal unique, les méthodes de chronométrie mentale (additives ou soustractives) ont permis de mesurer le temps de traitement des informations. La rapidité du traitement a indexé les compétences cognitives de l'expert.

Les recherches issues de ce courant de recherche ont porté sur des compétences spécifiques développées par les experts dans leur domaine d'excellence (par exemple sportif). Elles ont traité de la perception ou de la décision. La centration sur les experts a été liée à la capacité de ces derniers à réaliser très régulièrement des performances élevées, dans des situations appartenant à leur domaine d'expertise. L'expert est considéré comme un modèle de performance. Les variables manipulées dans des situations standardisées sont censées être révélatrices des processus mobilisés par l'expert dans le domaine en question : elles présentent un rapport étroit avec le domaine d'expertise considéré, tout en le " disséquant », pour mieux contrôler les conditions expérimentales. Les questions sous-jacentes concernaient la nature des compétences de l'expert et les processus mobilisés. Elles ont été traitées à travers une série d'études non exhaustives liées à des domaines aussi variés que le sport, les échecs, le service (restauration), le diagnostic médical... Les résultats étaient consistants.

Qu'est-ce qu'un expert?

Il est difficile de définir l'expertise. Les travaux auxquels nous avons fait référence ont spécifié l'expert au regard de la performance, ils ont sélectionné des sujets experts pour les étudier, en dehors de la vie réelle. Ericsson (1996) et Abernethy (1993) ont montré que la caractéristique essentielle de l'expert consistait en sa capacité à réaliser des performances exceptionnelles dans un domaine particulier. Ces performances étaient reproductibles dans des conditions similaires (Ericsson \& Smith,1994 ; Ericsson \& Lehman, 1996). Elles n'étaient pas liées au hasard mais plutôt à des composantes personnelles relativement stables. L'expertise est apparue comme une hyper adaptation à la tâche (au plan des processus cognitifs et psychologiques). Elle n'était pas innée et la pratique a été déterminante dans l'adaptation aux contraintes de la tâche (Ericsson \& Charness,1994 ; Ericsson, 2002). Cette pratique a requis beaucoup d'efforts et les effets n'étaient pas observables immédiatement (Ericsson, Krampe \& Tesch-Römer, 1993). Elle était motivée par la volonté d'améliorer la performance et était indispensable à l'acheminement vers l'expertise ainsi qu'à son maintien. Une période préparatoire de dix ans était indispensable (et non toujours suffisante) pour obtenir des performances de niveau international (Chase \& Simon, 1973 ; Ericsson et al, 1993).

\section{L'étude des compétences de l'expert}

Différentes variables ont été étudiées : les compétences mnésiques, celles liées à la perception des situations, au raisonnement et à la mémoire... Nous avons présenté un ensemble de travaux, sous forme de tableaux, à partir de trois critères : la perception, la mémoire et le raisonnement. Nous avons tenté, dans la mesure du possible, de retenir prioritairement des travaux liés au sport. 
Les travaux sur la perception (recherche visuelle)

Ils visaient à décrire les caractéristiques distinctives des sujets à un niveau très local. Les études sont synthétisées dans le tableau 1.

Insérer le tableau 1

Les experts ont obtenu de meilleures performances que les novices dans les tâches proposées : ils étaient plus rapides et prenaient d'avantage d'informations pertinentes que les novices. Dans les études sur la détection de cibles, l'habileté perceptive était plutôt décrite comme une recherche visuelle spécifique à la cible et au ballon. Les habiletés d'anticipation étaient supérieures chez les experts, qui présentaient un avantage cognitif clair : ils traitaient rapidement les informations pertinentes des situations expérimentales construites, tout en ignorant les informations non pertinentes. Ils disposaient d'informations signifiantes qu'ils stockaient en mémoire à long terme et qu'ils rappelaient de façon efficace (Ericsson \& Chase, 1982).

Ces travaux ont permis d'évaluer les mécanismes cognitifs à l'origine de la performance. Les tâches expérimentales ont été «définies », en référence aux travaux de Simon (1973) : les buts, les contraintes et les opérations à mettre en œuvre apparaissaient clairement aux individus. De manière usuelle dans les approches expérimentales, les tâches sont standardisées: elles sont identiques pour tous, les variables sont contrôlées par l'expérimentateur. Les variables mesurées sont quantitatives : elles correspondent au taux d'erreur (ou à la précision des réponses : nombre de situations, de pièces, de joueurs rappelés avec précision) et au temps de réaction. Ces études ont amené à s'interroger sur la modélisation et sur la simulation des tâches au regard des situations rencontrées dans le monde du travail. Ces études ont permis d'identifier des compétences spécifiques de l'expert au regard de celles du novices, mais elles n'ont pas pu rendre compte de son activité. Elles ont produit des connaissances "statiques » qui ne renseignaient que partiellement sur l'activité dynamique des sujets en situation. Van Daele (1997), en psychologie du travail, a insisté sur la différence entre des situations standardisées, des situations simulées (qui tentaient de reproduire les situations issues de la pratique) et des situations naturelles (qui correspondaient à la pratique elle-même). Si chacune présentait un intérêt particulier, elles ne pouvaient être assimilées et les résultats ne devaient pas être généralisés des unes vers les autres.

Les experts étudiés étaient différents : certains avaient un niveau national ou international (Allard et al, 1980...), d'autres étaient présentés à partir d'une durée de pratique (Garland \& Barry, 1991), ou d'un niveau de connaissances (Chiesi, Spillich \& Voss, 1979). On s'est alors interrogé sur les éléments qui amenaient ces auteurs à choisir leurs experts. La définition de l'expertise sensée être acceptée par tous, n'était pas consensuelle, elle a différé entre des situations expérimentales et des situations de la vie quotidienne.

Depuis quelques années, d'autres études ont été développées dans le domaine sportif : elles ont visé l'amélioration de la perception de l'expert (recherche de stratégies visuelles avec l'équipe d'Abernethy en Australie), la compréhension des liens existant entre la perception et certaines émotions (avec l'équipe de Williams en Grande Bretagne), le développement de l'expertise (équipe de French et de McPherson aux Etats-Unis). Ces derniers travaux n'ont pas été rapportés dans la mesure où l'enfant ne pouvait pas être considéré comme expert, en référence à la règle des dix ans de pratique intensive (Chase \& Simon, 1973). 
Les travaux sur la mémoire

Les études visaient à comprendre la façon dont les experts classaient les problèmes et à évaluer le rôle des connaissances dans la réalisation des tâches (voir tableau 2).

\section{Insérer le tableau 2}

Les performances des experts étaient supérieures dans les tâches mobilisant les habiletés liées à la mémoire. Ericsson et al $(1980,1988)$ ont insisté sur l'idée d'une mémorisation catégorielle des items, à travers laquelle ces derniers seraient classés avant d'être mémorisés. Jusqu’à présent, la mémorisation était considérée comme étant "perceptuelle », c’est-à-dire répondant à l'ordre de présentation des items. Les tâches proposées au sein des expérimentations étaient standardisées, les mesures étaient quantitatives (taux d'erreur et temps de réaction). Deux des trois recherches étaient éloignées de la réalité quotidienne. La deuxième (Ericsson \& Polson, 1988) se rapprochait de la pratique. Les auteurs l'ont considérée comme étant plus difficile que la situation de référence, dans la mesure où le serveur devait associer des plats avec des photos, plutôt qu'avec des personnes réelles. Les études rapportées portaient prioritairement sur des domaines d'expertise non liés au sport, ils ont été largement développé par l'équipe d’Ericsson. Le niveau d'expertise des sujets différait: 12 ans de pratique pour l'étude de Debû et al (2003) et une expérience professionnelle affirmée pour l'étude d'Ericsson \& Polson (1988). La première étude (Ericsson et al, 1980) n’a pas fait appel à des experts, mais elle a constitué une étude pionnière sur la mémoire.

\section{Les travaux sur le raisonnement et sur les bases de connaissances}

Les travaux princeps étaient liés aux échecs. D’autres études se sont développées dans des domaines de la physique, de l'informatique... Les paradigmes et la méthodologie ont été repris par la suite pour étudier les bases de connaissances des experts sportifs. Les études ont traité de l'expertise des athlètes et de celle des entraîneurs. La recherche avec les entraîneurs a permis de valider une méthodologie, utile pour l'étude des bases de connaissances des entraîneurs experts. Ces études ont été répertoriées au sein du tableau 3.

\section{Insérer le tableau 3}

Les travaux liés au raisonnement et à l'évaluation des bases de connaissances ont porté sur des domaines variés d'expertise. L'expérience de De Groot (1978) a été considérée comme pionnière : elle a utilisé des protocoles de pensée à voix haute qui étaient loin de faire l'unanimité. Une polémique tendait à opposer des chercheurs favorables à l'utilisation des verbalisations pour accéder aux processus mentaux (Ericsson \& Simon, 1980), et ceux qui s'y opposaient (Kellog, 1982 ; Nisbett \& Wilson, 1977). Rappelons que les années 1970 et le début des années 1980 ont été marquées en psychologie, par l’hégémonie des méthodes expérimentales : les chercheurs ont élaboré des hypothèses qu'ils ont testées à partir de tâches standardisées. Les données étaient quantitatives, on a mesuré des variables indépendantes en contrôlant la variable dépendante. Par la suite, trois types d'approches ont été utilisées : des approches qualitatives, des approches quantitatives et des approches mixtes. Les méthodes de recherche quantitatives se sont développées dans les sciences naturelles pour étudier les phénomènes naturels ; les méthodes de recherche qualitatives se sont développées dans les sciences sociales, afin de permettre l'étude des phénomènes sociaux et culturels, dans le contexte de vie des individus. La théorie ancrée de Strauss \& Corbin (1990) dont il a été fait référence dans les travaux de Côté et al (1995a, b et c), est une méthode de recherche qualitative. Les hypothèses n'étaient pas posées au préalable (comme dans les méthodes 
quantitatives), elles ont émergé de l'analyse des données. Cette méthode a été ascendante : on est parti des résultats pour élaborer des hypothèses que l'on a vérifié à travers une analyse plus fine. Il n'y avait ni variable dépendante, ni variable indépendante. Les travaux de Strauss \& Corbin (1990) ont porté sur l'étude du milieu hospitalier. Le chercheur a accordé de l'importance aux verbalisations des individus et leur position s'est vue modifié : l'individu n'était plus « sujet d'expérimentation », il est devenu « participant », voire " acteur » : ses propos ont pu influencer le cours de l'expérience dans un sens non connu de l'expérimentateur. Ce dernier n’a pas tout contrôlé (il n'a pas tenté de le faire). La notion d'expérience a pris le pas sur celle d'expérimentation, au sens positiviste.

Les travaux rapportés dans cet article ont utilisé les deux types de méthodes ; ils ont mis en évidence des données quantitatives (temps de réaction, taux d'erreur) ou qualitatives (nature des connaissances rapportées...). Les sujets ou participants ont fait l'objet des mêmes réserves que ceux des études précédentes : leur niveau d'expertise n'était pas connu. Au plan sportif, les études ont porté sur l'expertise des athlètes, mais aussi des entraîneurs : les études de Côté et al (1995a, 1995b, 1995c) étaient qualitatives, elles ont été pionnières dans ce domaine. Elles ont montré que l'expertise des entraîneurs pouvait être étudiée avec des méthodes similaires à celles utilisées pour l'athlète. Il a pu paraître surprenant au lecteur, de traiter de l'expertise de l'entraîneur, mais les métiers du sport concernent à la fois les professions d'athlète et d'entraîneur.

Dans les études sur la planification et le raisonnement, les représentations des experts sont apparues plus abstraites que celles des novices, les experts s'intéressaient davantage à la solution qu'à l'énoncé lui-même, ils avaient davantage de connaissances et celles-ci étaient globalement plus structurées. Les études sur l'évaluation des bases de connaissances des experts ont montré l'importance des connaissances et des raisonnements tacites dans la résolution de problèmes.

Toutes ces recherches se sont plus ou moins directement acheminées vers la construction de modèles locaux de mémoire, de raisonnement. Elles ont été intéressantes pour extraire l'information de domaines mal définis ou flous, dans lesquels certains aspects de la situation critique n'étaient pas clairement spécifiés. Toutefois, elles n’ont pas permis de comprendre les processus cognitifs mobilisés par l'expert en situation complexe inhérente au domaine d'expertise.

\section{Interrogations sur les modèles qui sous-tendent ces recherches}

Les recherches ont tenté de rendre compte des processus cognitifs caractérisant l'expertise dans un domaine spécifique. Au total, chaque étude s'est centrée sur un type de processus ; il a été difficile de dégager de ces résultats, une vision des compétences cognitives " globales ». Dans les situations expérimentales, les chercheurs ont écarté le contexte, afin de contrôler au maximum les variables indépendantes. Cette mise à l'écart du contexte a amené à s'interroger sur la cognition décrite de cette façon, au regard d'une cognition en situation « naturelle » finalisée par des objectifs professionnels. Le concept de tâche a été questionné : il a semblé être à l'origine d'un premier niveau d'analyse du travail, qui était général et simplificateur. En sport, la tâche n'a pris en compte ni la nature du rapport de force, ni les compétences propres des athlètes, ni la complexité de la situation, ni la fatigue, ni les émotions, ni le bruit, ni l'éclairage... Ces variables personnelles et environnementales auraient été considérées comme des variables parasites qu'il aurait fallu neutraliser. Cette tendance à l'abstraction pour analyser les perceptions et les capacités motrices a été dénoncée par Varela (1989a). Selon lui, elle a été symptomatique d'une partie des sciences cognitives. Elle n’a pas permis de comprendre l'intelligence cognitive, dans la mesure où cette dernière ne pouvait se concevoir que dans son intégration corporelle (« embodied»). Les individus étaient considérés comme des agents incarnés, couplés en permanence avec leur contexte. 
Le contexte consiste en l'environnement physique et humain de la situation, en son histoire (les événements précédents), en l'enjeu ou les enjeux pour les acteurs, en les compétences et en l'état de fatigue et de motivation de chacun... Avec le concept de tâche, on a su " ce que devait faire l'individu », mais on ne savait pas comment il devait s'organiser pour le faire. On s'est intéressé davantage aux résultats de l'action qu'à « l'activité ». Leplat \& Hoc (1983), ont considéré l'activité comme réalisation (la tâche a été appréhendée en tant que prescription d'objectifs et de procédures). Clot (1998) l'a définie au regard de la tâche qui la « déclenche », elle est liée au système fonctionnel individu-tâche. Amalberti \&Hoc (1998) ont tenté de comprendre comment l'individu s'était organisé pour réaliser un travail, en fonction de normes de production et de procédures, "plutôt que de chercher la correspondance stricte entre consigne et exécution » (p. 12). Clot (1998) a considéré l'activité comme une triade vivante tournée vers son objet et vers l'activité d'autrui portant sur cet objet. Il a insisté sur le caractère social de l'activité (statut de l'environnement et statut de la cognition).

La focalisation sur l'activité a impliqué une prise en compte de la situation et non plus seulement de la tâche. La situation a été conçue par Amalberti \& Hoc (1998) comme le couple opérateur /outil / contexte à chaque instant ; elle ne s'est pas réduit à l'intention de l'opérateur, protégée des intentions concurrentes (comme la tâche). Lave (1988) a décrit deux composantes de la situation : l'arena et le setting. La première renvoyait à l'environnement spatial et social objectif : elle présentait la situation comme donnée. La seconde concernait la partie de l'environnement qui était marquée par l'action : la situation apparaissait comme le produit de l'activité. La tâche prescrite aurait consisté en l'arena, alors que le setting aurait plutôt été lié à la situation.

\section{Conclusion}

Les recherches ont poursuivi deux objectifs. Le premier renvoyait à la construction de modèles de compétences (mobilisées par les experts en situations standardisées). Le deuxième consistait en l'élaboration d'une théorie des tâches : les tâches pourraient être construites et identifiées selon les compétences mobilisées et selon leur niveau de mobilisation. Famose (1990) a tenté de construire une telle théorie, en s’appuyant sur les travaux de Fleishman \& Quintance (1984) sur les aptitudes. Il a pu sembler illusoire de se satisfaire d'une théorie des tâches ou de modèles de compétences, pour expliquer les fondements de la performance de l'expert. Une réflexion sur la notion de contexte (dans son caractère dynamique et dans son couplage à l'individu) a permis d'envisager la cognition sous un autre jour.

\section{La controverse : une prise en compte différenciée du contexte}

La controverse s'est développée dans différents domaines des sciences humaines. Dosse (1995), a montré comment des courants ont pu se développer et se croiser partiellement (sociologie, éthnométhodologie, psychologie du travail, ergonomie...). Ces réflexions ont été reprises et poursuivies dans le domaine du sport. Nous n'avons pas traité pas ici de l'ensemble de ce cheminement théorique et réflexif et nous nous sommes limités à l'étude de deux courants liés à l'ergonomie de langue française. L'ergonomie étudie l'activité de travail dans la perspective d'une meilleure adaptation du travail à l'homme ou de l'homme au travail (Daniellou, 1996). Deux cadres théoriques ont été utilisés. Le premier a correspondu historiquement à une centration sur le facteur humain des systèmes hommes - machines (plutôt dans les pays anglophones) et le second a renvoyé à une focalisation sur l'activité humaine (plutôt dans les pays francophones, mais cette approche tend à s'universaliser). Cette distinction a incité De Montmollin (1995) à envisager le pluriel pour ce concept et parler ainsi d'ergonomies. 
La controverse est née de la différentiation du rôle et de la place accordée au contexte, dans l'analyse de l'expertise. Pour Leplat (2000), la notion de contexte est apparue ambiguë dans la littérature. Contexte et environnement ont souvent été synonymes, ils ont exprimé les circonstances et les conditions de production d'une action, d'un événement. Schématiquement, deux façons d'envisager le contexte ont pu être distinguées. Dans la première, le contexte a été peu ou pas pris en compte, dans la seconde, il l’a été largement.

\section{La faible prise en compte du contexte}

La faible ou l'absence de prise en compte du contexte s'est observée à travers les « modèles classiques » de l'étude de l'expertise. L’environnement a été considéré au mieux, comme une variable indépendante ; il n’a pas été pris en compte dans sa globalité, mais simplement dans certains aspects jugés pertinents par les expérimentateurs (exemple: caractère structuré ou non des situations observées par les joueurs, nombre de joueurs, temps disponible pour agir, support de la situation -diapositive / film / tableau magnétique... ). Ces modèles se sont inscrits dans le cadre des approches expérimentales : le chercheur tente de contrôler un certain nombre de variables, dont certaines sont liées au contexte. Dans ces modèles classiques (liés aux approches cognitives classiques), l'environnement est envisagé à partir d'une conception déterministe : le contexte perçu par le sujet est censé être identique à celui qui est conçu et perçu par l'expérimentateur ; le sens accordé à l'environnement est présumé similaire entre les différents individus impliqués dans l'expérimentation. Ces "modèles classiques » se sont appuyés sur des approches déterministes et causalistes de la cognition et de la performance: telle situation est sensée être interprétée d'une façon particulière et déclencher chez les sujets des comportements appartenant à un registre spécifique. Ceux-ci peuvent être évalués à travers la performance (variable dépendante).

La notion de contexte a été largement affaiblie: le contexte est apparu à travers la tentative de prise en compte des conditions de la pratique quotidienne dans la situation expérimentale. Mais la situation expérimentale a différé de la situation de référence (exemples : situations d'observation de diapositives, de film, en référence à une situation de compétition sportive). Le contexte a ici été banalisé, il a été considéré comme une variable pouvant influencer la performance, au même titre que d'autres variables. Les études qui sont liées à ces « modèles classiques » ont été développés dans la première partie de cet article.

\section{La réelle prise en compte du contexte}

Une seconde façon d'envisager le contexte a reposé sur sa réelle prise en compte. Il a été considéré comme étant en partie à l'origine des actions (les composantes individuelles liées aux compétences, aux motivations, aux attentes, aux buts poursuivis influençaient également ces actions). Il a permis de donner du sens à la situation (exemple situation de compétition ou d'entraînement), en même temps qu'il a pu être significatif pour l'individu (importance accordée à cette compétition). La cognition n’est plus apparue sous une forme déterministe, mais sous une forme "opportuniste» (Sperber, 1996): elle varie en fonction de l'environnement de l'individu, elle est ancrée dans le monde. La cognition est considérée comme étant située dans l'espace et dans le temps pour un individu. Elle est singulière et contingente au contexte.

Plusieurs façons de prendre en compte le contexte ont été envisagées dans la littérature. Le contexte a pu être considéré à travers la notion de situation (approches de la psychologie cognitive du travail ou de l'ergonomie cognitive), il a pu être étudié à travers son couplage avec l'individu (approche de l'anthropologie cognitive située), ou encore à partir de son caractère organisateur de l'action à travers la construction du sens (théories de l'action qui ont une origine largement sociologique). Seules les deux premières approches ont été analysées dans l'article : elles ont proposé des postulats et des méthodologies de recherches qui ont fait 
l'objet de recherches sur l'expertise en sport. La troisième "voie » a été liée à des courants variés, développés par Dosse (1995) ou par Lahire (1998).

\section{Le contexte considéré à partir du concept de situation}

L'ergonomie cognitive a développé une approche à partir de travaux sur l'analyse de l'activité dans le pilotage d'avions de chasse, la conduite de centrales nucléaires, de sous marins nucléaires... (Amalberti, 1996 ; Valot, 1996 ; Cellier, 1996 ; De Keyser, 1997...). Dans cette approche, l'environnement a été composé de situations. Pour Leplat \& Hoc (1983), le concept de situation a permis de passer d'un espace virtuel (la tâche), à un espace réel (le contexte de la pratique). La situation englobe l'homme alors que la tâche lui est extérieure. La situation est dynamique : elle évolue avec l'activité de l'individu, qui se modifie en retour. Deux types d'évolution ont pu être distingués à la suite de De Keyser (1988) : une évolution exclusivement en rapport avec l'action de l'individu (exemple du saut en hauteur) et une évolution liée à l'action de cet individu, à l'action d'autres individus, ou à un changement du milieu (régate en voile). Cette évolution différenciée a permis à Amalberti \& Hoc (1998) de distinguer les situations statiques des situations dynamiques: les premières n'ont qu'une source possible d'évolution, ce qui n'est pas le cas des secondes. En situation dynamique, l'action présente un caractère irréversible, ce qui n'est pas toujours vrai en situation statique (une opération peut permettre de revenir à l'état initial) ; une non action peut modifier le problème à résoudre (Cellier, 1996).

Amalberti \& Hoc (1998) ont envisagé le contexte à partir des paramètres de contrôle, d'incertitude et de temporalité.

Les situations évoluent au cours du temps. On a pu distinguer deux aspects : la durée disponible pour agir (au delà d'une certaine durée, l'individu ne peut plus agir : effet butoir) et l'évolution dans le temps d'une partie de la situation (appelée "processus »). Ainsi, au volley-ball, l'effet butoir renvoie au rebond du ballon qui arrête la séquence de jeu; l'évolution du processus est liée à la prise d'appel de plusieurs attaquants en des lieux et des instants différents, la position du contre... Deux types de stratégies ont pu être adoptés pour faire face aux fluctuations du processus (Cellier, 1996). La première était réactive : l'individu s'est adapté en cours d'action à l'évolution du processus (attente du ballon). La seconde était anticipative : elle a consisté à prévoir l'évolution du processus de façon à anticiper les actions requises, le moment de leur mise en œuvre, ainsi que leurs conséquences probables sur le processus, afin de tenter de le conserver dans des limites acceptables (on ne peut pas réellement le contrôler). Cette anticipation a contribué à diminuer la complexité de la situation; elle a représenté un moyen de gérer les ressources individuelles et collectives. L'anticipation pouvait porter, d'une part, sur les effets de l'action de l'acteur ou sur ceux de certains événements (incidents), et d'autre part, sur l'instant d'occurrence des actions, afin que les objectifs puissent être atteints (Van Daele \& Carpinelli, 1997). L'anticipation a largement pris en compte le contexte de la situation dynamique (présente, passée et projetée) : elle a été considérée comme une activité de régulation des différentes temporalités.

L’incertitude est considérée comme un élément omniprésent en environnement dynamique. Ses origines renvoient à la difficulté à identifier les événements probables, à déterminer l'instant de leur apparition, à délimiter leur lieu d'occurrence, à prévoir l'efficacité des réponses et leur influence sur le contrôle de processus... La fonction de l'opérateur consiste à s'adapter aux variations de l'environnement, afin d'atteindre le but fixé par le système, en fonction d'astreintes et d'attentes sociétales. Dans la littérature, l'incertitude a souvent été envisagée dans des études avec les pilotes, en particulier les pilotes d'avions de combat (Amalberti \& Deblon, 1992 ; Amalberti, 1991 ; Valot, 1996...). Elles ont insisté sur la nécessité d'une phase préalable de diagnostic (avec identification des solutions possibles) et 
sur la limitation de la durée de validité de ces solutions (notion d'échéance butoir). La décision efficace a été exécutée des conditions temporelles favorables, en fonction de savoirs faire disponibles.

Le contrôle concerne l'incertitude, la temporalité et les risques. Les deux premières directions du contrôle renvoient à ce qui précède (l'individu tente de gérer l'incertitude et le temps). La troisième concerne la gestion des risques liés à l'échec, à l’intégrité corporelle... Le risque influe sur le stress et sur la décision (perception très partielle de la situation, décision précoce qui ne prend pas en compte l'évolution du processus...). Dans sa décision, il doit réaliser en permanence un compromis entre la prise de risque, l'efficacité de ses actions et ses ressources propres. Valot (1996) a envisagé un « effet toboggan » lorsque le pilote ne disposait pas du temps nécessaire pour anticiper les événements probables. Amalberti \& Deblon (1992) ont montré qu'il tendait à élaborer des décisions pauvres lorsqu'il était acculé par le temps. Pour Amalberti (1991), il devait parvenir à ajuster les risques en fonction des compétences requises par la situation, de ses compétences actuelles et du temps disponible, dans le but de gérer ses propres ressources. Ce contrôle nécessitait des activités cognitives variées qui pouvaient être conscientes et réfléchies ou automatiques. Les activités étaient à l'origine d'une multiplicité de buts qui pouvaient être ou non compatibles pour l'opérateur, ainsi que pour les autres intervenants de la situation (gestion permanente du compromis entre coût et efficacité). Amalberti \& Hoc (1998) ont insisté sur l'activité de l'individu : la situation dynamique ne peut être conçue qu'à travers son couplage avec l'acteur, considéré dans sa complétude et dans son histoire.

Dans de nombreuses activités sportives, le contrôle de la situation n'est pas total, il ne concerne le plus souvent qu'une partie de la situation (processus) : il constitue un objectif pour l'acteur. Van Daele (1997) a montré qu'il était inhérent à la complexité de la situation. Celle-ci dépendait de facteurs externes (l'incertitude, la temporalité et le contrôle) liés à la dynamique de la situation et de facteurs internes qui interagissaient. Ces derniers renvoyaient: aux connaissances construites par l'individu sur la situation et sur son environnement (qui lui permettait d'anticiper les événements à venir), aux compétences de l'individu dans le domaine d'actions considérées, à ses connaissances sur sa propre cognition et à ses limites dans la situation actuelle, aux procédures qu'il pouvait mettre en place, à ses méthodes de travail, ainsi qu'aux métaconnaissances qui lui permettaient de s'auto-évaluer.

Dans ce cadre de pensée, on a considéré qu'une situation était complexe pour quelqu'un. La complexité n’était pas a priori, même si certains éléments y ont contribué. Elle a été mise en évidence à travers l'interaction entre des facteurs externes et des facteurs internes. La complexité et les compétences ont semblé entretenir un rapport de co-détermination. L'acteur devait en permanence ajuster ses possibilités aux exigences perçues de la situation. En volleyball, dans une situation de réception de service, le réceptionneur doit ajuster ses compétences à lire la trajectoire, à se placer et à réceptionner le ballon aux composantes spatio-temporelles de la trajectoire du ballon.

Enfin, la complexité pouvait être distribuée : elle pouvait se répartir entre l’individu et les dispositifs du système placés dans l'environnement, tels les partenaires et les adversaires en sport collectif.

On a insisté sur l'analyse de la situation par l'acteur, à partir de la perception de certains éléments, qui ont contribué à la fois à lui donner du sens et à réduire la complexité. Pour Hollnagel (1993), qui a développé un modèle du contrôle de l'action par le contexte, les actions n'avaient de sens qu'à travers le contexte qui les a vues naître. Le contexte était à la fois porteur de sens et donnant du sens. Il a orienté l'exécution de l'action. 


\section{Contexte et individu : l'histoire d'un couplage}

Une deuxième façon de prendre en compte le contexte a consisté à le considérer à travers son couplage à l'individu. Cette approche a été développée par Varela (1989a), en biologie, à partir de réflexions philosophiques. Elle a été utilisée par Theureau et son équipe en ergonomie cognitive et dans le domaine du sport avec Durand et ses collaborateurs. Elle a trouvé ses fondements dans le courant de la philosophie pragmatique, avec la réflexion de Peirce (1978) et dans celui de la phénoménologie, en particulier avec Merleau-Ponty (1945). Le pragmatisme est une théorie de la signification d'un terme ou d'une proposition en fonction de l'ensemble des effets qu'il produit. Le pragmatisme s'intéresse à l'action. Pour Peirce (1978), l'action n'est jamais terminée, elle est incarnée, c'est-à-dire qu'elle est incorporée à l'organisme qui en est à l'origine. On parle d'action de quelqu'un à un instant : elle a une temporalité, une singularité et une contingence. Elle ne peut être séparée de la cognition vécue qui permet de lui donner à la fois une intention et un sens. La cognition est également incarnée, elle dépend d'expériences vécues dans un contexte à la fois biologique, psychologique et culturel plus large. On considère que la cognition reste inaccessible tant qu'elle n’est pas envisagée dans son fondement social.

Dans le cadre de l'interaction pragmatique, trois niveaux d'analyse sont interpénétrés. Le premier niveau consiste en la relation du sujet connaissant avec son contexte. Elle est à l'origine d'une rationalité au regard de règles techniques dans le travail. Le deuxième renvoie à la relation du sujet moral qui agit dans le monde social à partir de normes particulières et acceptées. La troisième est liée à la relation du sujet par rapport à sa propre subjectivité et par rapport à celle d'autrui. A ces trois niveaux, on peut envisager une dimension réflexive : le discours ne peut être réduit au langage ou à l'intention, il les dépasse, incluant par là même le monde vécu, l'histoire de l'individu, les actions. Ces quatre dimensions permettent de prendre en compte l'individu avec le contexte à la fois social, professionnel, individuel et temporel. Elles permettent de comprendre ce qui fait sens pour un individu engagé dans une situation à un instant. Merleau-Ponty (1945), a insisté sur le rapport indestructible entre l'individu et son environnement. Ce rapport ne peut être abordé que subjectivement en fonction de l'expérience et de l'intentionnalité propre du sujet. La prise en compte du contexte est apparue à travers le couplage entre l'individu et l'environnement, mais aussi la temporalité, l'intentionnalité...

Varela (1989a) a postulé l'existence d'une interaction circulaire entre l'individu et son environnement, qui restent autonomes l'un par rapport à l'autre. S'ils sont couplés l'un à l'autre, ce couplage n'est jamais définitif, il peut être remis en cause à tout moment, un autre couplage peut apparaître. Ce couplage est rendu possible par les capacités sensori-motrices de l'individu, qui sont utilisées à un instant dans un contexte à la fois biologique, psychologique et culturel. La construction du sens va apparaître à travers leurs déterminations réciproques : l'individu va donner du sens à l'environnement en même temps que l'environnement va susciter du sens pour lui. Le monde n'est plus envisagé dans ses aspects fondamentalement physiques, mais plutôt sous une forme fondamentalement sémiotique : il contient des signes qui vont aider à la donation de sens par l'individu. Ces signes sont perçus ou non par l'individu, en fonction de son histoire. On observe un déplacement du monde physique vers le monde vécu, expérientiel et significatif pour l'individu. L’environnement n’est plus considéré dans ses aspects physiques comme étant prédéterminé, il apparaît plutôt sous une forme sémiotique, en rapport étroit avec le vécu de chacun. La perception du monde et le sens accordé par chacun dépendent ainsi de l'histoire de l'individu. Perception et sens ne sont plus prédéterminés et uniformes, ils sont pluriels et dépendent directement de la nature des interactions circulaires qui unissent l'individu à son environnement. Selon l'intuition de Varela (1989a), la faculté humaine la plus importante renvoie à la possibilité de poser des questions pertinentes qui surgissent à chaque moment de notre vie. Elles ne sont pas prédéfinies mais énactées : on les fait émerger d'un arrière plan de la conscience, d'une 
manière toujours contextuelle. Dans cette perspective, action et signification sont indissociables, elles proviennent du couplage entre l'individu et son environnement.

La cognition et l'action s'inscrivent dans une temporalité, elles sont liées aux expériences vécues. L’idée fondamentale de Varela (1989a) a consisté à considérer les capacités cognitives comme intimement liées aux différentes expériences vécues. La cognition apparaît comme un "faire émerger ", qui devient créateur du monde dans un but plus large d'adaptation à ce monde. La cognition est incarnée, elle dépend des expériences vécues par l'individu, qui s'inscrivent dans un contexte à la fois biologique, psychologique et culturel qui le dépasse. Pour Varela (1989a), l'approche de l'énaction a visé à prendre en compte la temporalité de la cognition, c'est-à-dire l'histoire vécue par l'individu au sein de sa culture. Cette approche a considéré les dimensions ontologique, phylogénique et culturelle de l'individu couplé à son environnement à un instant (Varela, Thompson \& Rosch, 1993). Ces derniers ont insisté sur la prévalence du vécu, de la temporalité et de l'attrait pour la nouveauté : si le passé oriente les perceptions et les cognitions de l'individu, le présent, dans ses aspects inédits va faire signe à l'individu, qui va tenter de lui donner du sens pour agir. On a insisté sur l'idée d'un présent cognitif qui est à la fois intime (dans sa singularité et sa contingence et dans ce qui va contribuer à donner du sens), social (l'individu est couplé à un environnement social) et qui se poursuit sans cesse. Le présent est également fondamental pour coordonner les acteurs engagés dans un même environnement; ce sont les significations accordées à chaque instant pour chacun qui vont contribuer à déterminer le sens de l'action.

Nous avons vu que le sens émergeait du couplage entre l'individu et l'environnement : il a été suscité par l'environnement (à partir des signes) en même temps qu'il a été " donné » par l'individu. La signification a émergé à partir d'une intersubjectivité entre les acteurs et de leur compréhension réciproque. La signification est liée à une activité d'interprétation permanente et à un souci de compréhension d'un domaine d'action (Varela et al, 1993). Le sens donné à l'événement n’était pas prédéterminé, il était lié à un processus émergent qui s'est construit dans la durée qui était fonction de chacun. Il était inhérent à la perception._Pour Varela et al (1993), « la perception consiste en une action guidée par la perception » (p.235). En d'autres termes, il s'est agi de comprendre comment l'individu guidait son action de perception de l'environnement et comment ou par quoi elle était orientée. La façon dont l'individu est inscrit dans un corps détermine ses moyens d'action et de compréhension. La perception est directement liée au monde, elle contribue aussi à l'émergence des significations de ce monde environnant. Voir ne consiste pas en l'extraction des traits visuels, mais en le guidage de l'action dirigée vers eux. Comme Gibson (1979), ils ont rejeté la conception représentationniste de la perception fondée sur la notion de monde préétabli, à travers laquelle nous extrairions tous les mêmes éléments jugés pertinents de l'environnement. Gibson a refusé toute forme de dualisme entre le sujet et l'objet. Mais à sa différence, la perception a été conçue comme une action guidée par la perception. La théorie de Gibson a largement été fondée sur l'environnement, mais il a considéré l'individu indépendamment de l'environnement. Ce dernier a été décrit par rapport à ce qu'il y avait à percevoir, en fonction d'un organisme mobile et actif. Les systèmes perceptifs seraient ainsi phyologénétiquement adaptés à la perception d'informations dans l'environnement. La perception a été considérée comme active, elle est apparue comme une combinaison entre la perception de l'environnement et la perception de soi. Les informations utiles seraient directement perçues par le sujet en fonction du registre d'actions permises dans un environnement particulier.

Dans la filiation à Merleau-Ponty, le sens est lié à l'expérience et à l'intention. L'intentionnalité renvoie à la fois à la façon dont l'individu interprète le monde et à la manière dont cette interprétation est adaptée au monde. En d'autres termes elle est inhérente aux possibilités d'action et à la manière dont les situations induites par ces actions satisfont ou non à ces possibilités. L'énaction est un processus qui ressemble à une dérive naturelle. Ce 
processus permet de cheminer pour trouver l'interprétation du monde à laquelle corresponde une action satisfaisante de l'individu couplé à son environnement. On a insisté sur le vécu et sur l'attrait pour la nouveauté ; l'objet privilégié est le présent cognitif, c'est-à-dire les cognitions actuelles de l'individu (qui s'appuient également sur des cognitions passées). Ce processus de « dérive naturelle » se distingue des programmes de résolution de problèmes, utilisés dans l'approche cognitive computationnelle. La résolution de problème induit la notion de problème connu, dont le sens est partagé par les individus. Dans le cadre de l'énaction, le sens émerge du couplage entre l'individu et l'environnement, il n'est pas prédonné et peut dans ce cas différer entre les sujets (même si la communication vise en partie à réduire les variations de significations accordées au contexte).

La construction du sens est rendue possible par une activité interprétative. A partir de l'éthnométhodologie, cette activité a pu être appréhendée à partir d'une analyse descriptive, voire réflexive de l'acteur en situation, qui a porté à la fois sur les actions et les procédures utilisées, mais aussi sur les circonstances qui étaient à l'origine de l'activité. L'individu peut rapporter ce qui a été qualifié par Garfinkel (1967) d' "accountable », c'est-à-dire observable, descriptible, intelligible, racontable et analysable. L' "accountability » est rationnelle : elle renvoie à des productions méthodiques et intelligibles en situation. Elle permet d'avoir accès aux modes de structuration des activités ordinaires et aux compétences des individus. Le sens est appréhendé à travers ces descriptions rationnelles, narratives, voire réflexives des actions par les participants. Mais le sens usuel des mots est souvent incomplet : c'est la référence au contexte qui va permettre de le préciser (notion d'indexicalité du langage).

Dans l'idée d'un processus de dérive naturelle, l'action n'est jamais terminée, elle se poursuit pour satisfaire au monde perçu et subjectif. Elle est dirigée vers une situation qui n'existe pas encore réellement, elle est en devenir en fonction de l'activité perceptive et interprétative de l'individu. La situation n'est pas fixe, elle n'est pas déterminée ; elle est conçue dans ses relations avec l'individu. Les situations locales se transforment en permanence à partir de l'activité perceptive et interprétative de l'individu. La situation est induite par le couple "événement - situation » : l'individu donne du sens à l'événement en même temps que l'événement suscite du sens pour l'individu. Cette interaction réciproque incite à passer de la subjectivité (de l'acteur) à l'intersubjectivité (de l'acteur et de l'événement au niveau du sens qu'il suscite chez celui-là et du sens « donné » de celui-là à celui-ci, en fonction des signes perçus et de l'histoire du sujet). La cognition permet de poser des questions pertinentes : elles sont énactées de l'arrière-fond de la conscience. Elle permet également de tracer le chemin pour parvenir à des actions satisfaisantes. Action et signification apparaissent indissociables.

Cette approche de l'énaction a été à l'origine de l'approche de l'anthropologie cognitive située (Theureau, 1992 ; Theureau \& Jeffroy, 1994). L'expert a été considéré comme expert du couplage (interprétation et satisfaction des moyens d'actions choisis). Dans l'approche précédente, il était envisagé dans son rapport entre l'efficacité de ses action et le niveau de compréhension de la situation. La différence essentielle entre les deux approches a reposé sur leur postulat : la première a réfuté l'idée de représentation et de fonctionnement du cerveau sur la base de computation de signes et de symboles, alors que la seconde s'est appuyée sur cette idée de fonctionnement computo-symbolique, avec toutefois un affaiblissement de la notion de planification (qui est alors globale avant d'être affinée au cours de l'action, à partir de l'évolution de la situation). Leur similitude a renvoyé à la conception d'une «action située » dans le temps et dans l'espace (qui prend donc en compte l'environnement). Suchman (1978) a élaboré ce concept dans le cadre d'un travail de confrontation avec des chercheurs en intelligence artificielle, qui visait à construire des robots capables d'agir de manière intelligente et autonome. Elle s'est heurtée à un modèle proche de celui de l'acteur rationnel. 
Selon ce modèle, l'individu produirait des plans d'action, avant d'analyser la situation occurrente et de choisir les moyens pour agir. L'action serait ainsi divisée en plans et en sousplans qui seraient ensuite exécutés. Elle a avancé l’idée selon laquelle l'élaboration préalable de plans précis ne pouvait faire l'économie de la prise en compte de l'environnement : l'action apparaît inséparable de la situation à laquelle elle est indexée. Suchman s'est intéressée à la façon dont l'individu inscrivait son activité dans un environnement. Elle s'est éloignée de l'idée du réglage de l'action par un plan préalable. Le plan ne servirait pas à guider ni à induire les différentes phases de l'action, mais plutôt à placer le sujet dans des conditions à l'origine d'une utilisation optimale de ses habiletés incorporées. Il permettrait de rendre compte de l'action, en formulant ses conditions et ses conséquences ; il constituerait une ressource pour l'action. La représentation de l'action a ainsi été appauvrie, ce qui a constitué un des buts de l'approche de l'action située, en réaction à un cognitivisme dur.

Le mode séquentiel de traitement de l'information proposé par Newell \& Simon (1972), n'a plus permis d'expliquer de façon satisfaisante la cognition en contexte (Sanders, 1986). La critique du modèle issu de l'intelligence artificielle s'est traduite, d'une part, par la remise en cause des approches basées sur la représentation symbolique de l'information et d'autre part, par l'appel au contexte, à travers le concept de cognition située. La critique d'un « cognitivisme dur », s'est caractérisée par deux aspects essentiels. Le premier a insisté sur le fait que la cognition est «opportuniste » : elle varie en fonction du contexte auquel elle est soumise (elle est située) et elle contient peu de planifications et de raisonnement. Le second a souligné le caractère « ancré » dans le monde ( « embodied »), l'environnement permet de guider le comportement à partir des contraintes, des repères et des aides pour l'action qu'il contient.

Le concept d'action située a induit celui de cognition située. Il a amené à s'interroger sur l'idée d'une connaissance totalement préexistante à l'action, qui serait activée en mémoire. Il a incité à s'interroger sur la possibilité d'une connaissance crée pour l'action et dans l'action, dans le cadre de l'interaction (circulaire ou non) entre l'individu et son environnement.

\section{Implications sur les concepts et conclusion}

La controverse est née de la place accordée au contexte. Elle a été à l'origine d'une construction nuancée du sens : dans un cas ce dernier est préétabli, dans l'autre, il est construit soit dans l'interaction entre un sujet et une situation, soit dans le couplage d'un individu à son environnement.

Ces conceptions différentes de la cognition et du rapport de l'homme au monde ont eu une incidence sur des concepts tels que la situation, le contexte, l'action, l'expertise. Ainsi dans l'approche cognitive classique, le contexte a été considéré comme préétabli, sa prise en compte n'est pas toujours apparue indispensable, ce qui a expliqué qu'il ait été si peu pris en compte. Dans les approches liées à la cognition située, le contexte a contribué à donner du sens aux actions, sur la base d'une activité d'interprétation de l'individu.

Le concept de situation a varié entre les trois approches présentées. Dans la première, la situation était expérimentale : elle comportait un but et des contraintes pour les sujets, ainsi qu'un ensemble de variables qui étaient contrôlées par l'expérimentateur. Cette situation était reproductible (selon un objectif majeur de la méthode expérimentale), et préétablie. Dans l'approche cognitive du travail (avec Amalberti, 1996; 2001 ; Amalberti \& Hoc, 1998 ; Cellier, 1996 ; Valot, 1996...), la situation était conçue dans le cadre de son interaction avec l'individu qui la réalisait. La situation a été inscrite dans un contexte qui l'englobait, tout en contribuant à lui donner du sens (notion de performance d'entraînement ou de compétition, selon l'enjeu accordé à la performance à réaliser : résultat ou maîtrise). Elle était singulière et contingente. Elle pouvait évoluer (elle était statique ou dynamique). Elle pouvait être décrite à partir de trois paramètres (l'incertitude, le contrôle et la temporalité). Dans l'approche de 
l'anthropologie cognitive située, qui s'est fondée sur la théorie énactive, la situation était locale, elle se transformait en permanence avec l'activité du sujet percevant, elle ne présentait aucune fixité.

Le concept d'action était inhérente au mouvement proprement dit, dans l'approche cognitive classique ou computationnelle. Dans l'approche cognitive « néocomputationnelle », il renvoyait à la partie de la conduite liée à ce qui précédait et ce qui suivait le mouvement (de la perception à la réalisation). Dans l'approche anthropologique située, l'action était dirigée vers un but, elle guidait la perception, mais aussi l'activité intersubjective d'interprétation du contexte... elle était attirée par la nouveauté.

Enfin, l'expert, dans l'approche cognitive classique, était celui qui obtenait très régulièrement des performances très élevées dans un domaine de compétences donné. L'expertise renvoyait à des compétences particulières, préalablement mises à jour. Dans l'approche " néocomputationnelle », l'expert était celui qui réalisait des actions satisfaisantes pour lui et adaptées à l'environnement (au plan de l'efficacité). Enfin, pour l'anthropologie cognitive située, l'expert était conçu comme expert du couplage : il parvenait à donner du sens au contexte qui faisait signe pour lui. Il existait alors un rapport de satisfaction entre son interprétation de l'environnement et les effets induits par cette interprétation sur l'environnement. Dans tous les cas, l'expertise est apparue spécifique au contexte, seule la conception du contexte et surtout de la situation a varié.

\section{Les modèles disponibles en ergonomie pour étudier l'activité de l'expert en sport}

Les modèles classiques ont analysé les compétences des experts et leur niveau de mobilisation dans leur confrontation à des situations expérimentales. Ils n'ont pas permis d'étudier l'activité de l'individu confronté à une situation (ce n'est pas leur but). Pour mesurer des compétences et comprendre leurs effets sur la performance, les chercheurs ont été amenés à construire des situations standardisées, dans lesquelles le contexte était banalisé. Or l'analyse de l'activité de l'expert n'a pu faire l'économie de l'utilisation de situations naturelles, elle n'a pu s'affranchir du contexte, qui est à la fois porteur de sens et donnant du sens. Il est apparu important de mettre l'accent sur l'analyse des interactions entre l'individu et son environnement, afin de mieux comprendre les significations de l'individu et son intention. En ergonomie de langue française, deux approches ont permis d'étudier l'expert en situation naturelle: le modèle de "la suffisance cognitive " avec l'équipe d'Amalberti et l'anthropologie cognitive située, développée par Theureau et utilisée dans le domaine sportif par l'équipe de Durand.

\section{L'anthropologie cognitive située : le primat de l'émergence}

L'anthropologie cognitive située a cherché à élaborer un modèle de construction de l'activité à partir du couplage entre l'acteur et son milieu. Elle a reposé sur l'hypothèse selon laquelle la cognition individuelle reste inaccessible, tant qu'elle n'est pas envisagée dans son fondement social, ou dans son couplage à l'environnement.

Les recherches sur l'énaction ont contesté radicalement l'idée de la cognition basée sur des représentations d'un monde extérieur prédéterminé. Elles ont accordé une grande importance au contexte, qui serait créateur de la cognition. Elles se sont appuyées sur l'hypothèse de l'autopoï̀se, élaborée par Maturana \& Varela (Varela, 1989b), à partir d'une réflexion théorique et épistémologique portant sur des études biologiques. Ils ont considéré que le phénomène central de la cognition est l'autonomie des systèmes vivants. Selon cette hypothèse, un système vivant « autopoïétéique est organisé comme un réseau de processus de production de composants qui : a) régénèrent continuellement par leurs transformations et leurs interactions le réseau qui les a produit et qui b) constituent le Système en tant qu'unité 
concrète dans l'espace où il existe, en spécifiant le domaine topologique (la structuration de cet espace) où il se réalise comme réseau » (p. 45). Selon l'intuition de Varela (1989b), la faculté humaine la plus importante est liée à la capacité à élaborer des questions pertinentes, qui émergent d'un arrière-plan de la conscience, en fonction du contexte. L'action et la signification de l'acteur sont inséparables ; elles sont issues du couplage entre l'acteur et son environnement : le couplage structurel.

La seconde hypothèse affirme que le système nerveux est opérationnellement clos : il dépend des processus interdépendants (notion de couplage). Le système ne peut pas différencier les perturbations qui sont issues de l'intérieur de celles qui proviennent de l'extérieur. La notion de perturbation remplace celles d'input / output (liées au cognitivisme). Le système se transforme de l'intérieur (il est autopoïétique) et non à partir d'éléments prédéfinis prélevés dans l'environnement. Les connaissances sont construites dans et pour l'action, elles sont locales.

Theureau ( 1992), dans une perspective d'anthropologie cognitive du travail a crée un objet d'analyse de l'action en cours (le cours d'action). Cette activité peut être racontée, montrée, commentée par lui-même à tout instant. Pour Theureau \& Jeffroy (1994), cet objet d'analyse devait permettre d'échapper au dilemme traditionnel lié à l'étude de la cognition humaine : solipsisme (la cognition est un processus interne à la personne) ou déterminisme environnemental (la cognition est guidée de l'extérieur). La perception a été considérée comme active (à la suite de Gibson, 1979) et l'action a pu avoir un effet à la fois sur l'état du couplage et sur la construction de connaissances. La communication a pu être conçue comme une construction commune d'informations plutôt que comme un échange d'informations. L’observateur s'est articulé avec l'acteur (il ne lui était pas extérieur).

La conception centrée sur le cours d'action a utilisé trois principes. Le premier concerne la globalité : il s'agit de considérer la situation de travail comme un tout, même si l'un des objectifs de l'étude du cours d'action consiste en la découverte de ses composantes. Le deuxième est lié à l'interdisciplinarité : il permet de coordonner les apports de différentes disciplines liées à la connaissance de l'homme au travail et cordonner cette connaissance à la conception technique et organisationnelle. Le troisième renvoie à la participation : il faut prendre en compte la subjectivité des opérateurs dans l'analyse et faire collaborer les opérateurs au recueil des données, à leur analyse et à l'élaboration des propositions de conceptions. Les opérateurs deviennent acteurs et non plus de simples exécutants d'une tâche standardisée.

Des études ont été menées dans le domaine du sport. Elles ont porté sur l'activité d'entraîneurs ou d'athlètes qualifiés d'experts, selon les critères de Côté et al (1995a). Elles se sont fondées sur le cadre méthodologique de l'analyse sémio-logique du cours d'action, élaboré par Theureau (1992) et détaillé dans son ouvrage. Les travaux sur l'activité des entraîneurs se sont inscrits dans un projet inter établissements déposé au Ministère des Sports, portant sur les connaissances et les pratiques des entraîneurs experts. Certaines études ont fait l'objet de publications d'autres sont plus modestement accessibles au sein d'un rapport.

\section{Insérer le tableau 4}

Ces études ont montré l'importance de la prise en compte du contexte par l'individu, dans l'organisation de son action. Les résultats ont indiqué : une différenciation des buts en fonction de la nature de la compétition (Saury \& Durand, 1997), des variations dans la programmation de l'entraînement selon le degré d'imminence des compétitions importantes (Sève \& Durand, 1999) et une différence dans le type d'activité privilégié par les pongistes au cours du match (composante exploratoire ou exécutoire, Sève et al 2002). L'action est apparue située dans le temps (D’Arripe-Longueville et al, 2001 ; Hauw et al, 2003) et dans 
l'espace (composé par les objets et les individus). Les individus engagés dans un cours d'action particulier, ont exploré, interprété sans cesse leur environnement afin de déceler des éléments porteurs de sens et de donner du sens à cet environnement. Donzé \& Durand (1987) ont insisté sur la prise en compte perpétuelle des caractéristiques individuelles de l'individu, à travers ses sensations physiques et mentales (notion de fusion entre le grimpeur et la paroi). En rapport avec les travaux de Suchman (1987), les planifications ont été adaptées au contexte, de façon opportuniste et émergente. L'action est née d'une activité interprétative basée à la fois sur l'utilisation de connaissances stockées en mémoire et sur la construction de nouvelles. L'exemple des pongistes était particulièrement révélateur : les connaissances sont apparues indissociables de leur engagement dans la situation actuelle ou passée.

Enfin, le choix des experts de ces études a répondu aux critères proposés par Côté et al (1995a) et Chase \& Simon (1973): dix ans d'expérience, un niveau de pratique ou d'entraînement élevé (international)... Ces critères étaient loin d'être utilisés dans toutes les études présentées jusque là.

Un second courant d'études a concerné une approche «néocognitiviste »: elle s'est appuyée sur la notion de représentation tout en limitant le rôle et la portée de la programmation de l'action.

\section{Le modèle de la suffisance cognitive : le primat de la représentation}

Un courant cognitiviste que l'on a pu qualifier «nuancé » a cherché à situer la performance dans son contexte d'apparition (situations naturelles voire simulées). Il a abandonné la conception de modèles cognitifs basés sur une hiérarchie de buts à atteindre, de type fin-moyens (Newell \& Simon, 1972); il s'est centré sur un «modèle de construction et d'actualisation constante de la représentation occurrente de la situation, soumis à un contrôle dynamique à partir de variables cachées » (Amalberti \& Hoc, 1998, p. 216). Il a tenté de comprendre la construction dynamique et circonstancielle des activités cognitives, à partir de l'idée d'interactions permanentes entre l'individu et l'environnement.

Ce courant a exploré les représentations, qu'il a considéré comme la couche sous-jacente de la cognition. Celles-ci ont pu être fortes et cohérentes, comme celles des patrons pêcheurs étudiées par Wisner (1996), ou pauvres et disparates. L'idée centrale était la suivante : le cerveau fonctionne sur des représentations et " la compréhension consiste à construire la bonne représentation du monde pour l'objectif visé » (Amalberti, 1996, p. 128). La notion d'intention, qui est apparue centrale dans les approches liées à la cognition située, est ici déterminante. Amalberti a insisté sur l'inévitable multiplicité de représentations possibles, en fonction des situations, des individus, de leurs intentions, de leur niveau de connaissances... Cette incommensurabilité des représentations a pu constituer un frein au partage de la compréhension dans une situation collective. Pour l'éviter et pour obtenir une compréhension congruente de la situation entre les individus, ils ont dû communiquer: partager des connaissances, des buts. On s'est éloigné de la notion de représentations d'un monde prédéterminé, telles qu'elles ont été conçues dans l'approche cognitive classique. Les représentations n'était pas uniques mais multiples et sans cesse actualisées.

L'approche de l'énaction a insisté sur la nécessaire construction de connaissances en cours d'action. Dans le courant de la suffisance cognitive, ces connaissances peuvent être construites en cours d'action ou stockées en mémoire à long terme avant d'être activées pour élaborer une représentation de la situation. Les situations de diagnostic médical ont requis l'activation en mémoire de schémas liés à des cas similaires à la situation actuelle (Raufaste, 2001). La précision du diagnostic dépendait de la richesse des connaissances des médecins, des faits pris en compte et des hypothèses qu'ils élaboraient à leur égard ainsi que de leur flexibilité face aux cas atypiques. Lorsque la situation était totalement inédite, la construction de la représentation ne semblait pas pouvoir se réaliser sur la base d'un raisonnement par 
« analogies » ou sur la base d'une typicalité, car il n'existait pas de représentations d'une situation analogue. La représentation de la situation occurrente devait alors s'élaborer à partir de l'agrégation progressive des informations perçues sur la situation et selon une intention particulière (raisonnement par abduction). La représentation (liée à l'activation en mémoire de représentations de situations similaires ou construite en cours d'action) a consisté en une simplification et une déformation du monde, à partir de l'objectif poursuivi et du niveau de connaissances de l'individu à cet instant. Ces représentations de la situation occurrente étaient toujours circonstancielles.

Le modèle de la suffisance cognitive contient l'idée d'un compromis nécessaire dans les possibilités de compréhension de l'opérateur en situation dynamique (Amalberti, 2001). Il s'est centré sur la « maîtrise de la compréhension », c'est-à-dire sur le nombre et la nature des incompréhensions que l'individu pouvait tolérer, dans la réalisation d'une performance. Il est basé sur la notion de "suffisance cognitive » qui répond à l'idée de marges fondamentales dans la gestion de la performance et du risque, afin de produire une réponse adaptée au contexte et satisfaisante pour le sujet. Il s'est appuyé sur deux variables : avant l'action, l'individu mobilise sa métacognition pour régler le compromis entre le risque accepté et la performance recherchée ; au cours de l'action, il cherche sans cesse des signaux de sécurité qui vont lui permettre de régler ce compromis de façon dynamique. D’autres réglages sont rendus possibles : ils utilisent des routines d'exécution qui intègrent leur propres niveaux de contrôle à partir d'émergences programmées (Amalberti, 1996). Ce modèle a concerné la notion de suffisance dans la planification, dans l'exécution et dans le contrôle de l'action (Amalberti, 2001). L'individu a élaboré un métaplan qui organisait globalement l'action, puis il réalisait des adaptations en ligne en fonction de l'évolution de la situation. La décision était donc composée de micro décisions plus ou moins satisfaisantes pour l'individu, qui, associées les unes aux autres aboutissaient à des résultats acceptables au regard des marges de la situation.

Ce modèle a permis d'envisager une gestion dynamique du couplage et une planification de l'action associée à des émergences programmées. Ce modèle de la suffisance cognitive a été développé dans les environnements à hauts risques : pilotage d'avions de chasse, conduite de centrale nucléaire, de sous-marins atomiques... En sport, la décision est irréversible, elle comporte une part de prise de risques pour l'individu (non mortel bien évidemment). Ces caractéristiques influent sur la nature de la décision à un instant, elles contribuent à modifier les marges acceptées par l'individu. La notion de programmation globale avec des émergences programmées a correspondu aux observations empiriques réalisées chez les volleyeurs : ils avaient une intention tactique et technique globale qu'ils ont peaufiné en cours d'action. Une étude (Macquet, 2001) a utilisé ce modèle pour étudier l'expertise des volleyeurs.

Insérer le tableau 5.

Les résultats ont montré que la compréhension de la situation n'était pas totale ; elle était juste suffisante pour agir en environnement dynamique. Cette compréhension s'est construite en cours d'action, à partir de composantes spatiales et temporelles qui pouvaient être disponibles assez tardivement. L'auteur a également mis en évidence que la performance des experts (selon des critères adaptés aux travaux de Côté et al, 1995a) n'était pas toujours exceptionnelle : leur maîtrise des situations était loin d'être totale (elle dépendait du rapport de forces entretenu entre les joueurs et entre leurs équipes).

Ces travaux issus de deux approches distinctes ont proposé un cadre théorique et méthodologique pour étudier l'activité de l'expert dans le contexte de sa pratique quotidienne. Ce cadre a permis de dépasser les limites des approches cognitives classiques, tout en 
présentant lui-même un frein lié à la «lourdeur» de l'expérimentation (conditions d'enregistrement, retranscription des entretiens, découpages des verbalisations et des situations de référence, traitement des données....). Ces protocoles ont été coûteux en temps, en matériel et en personnes, mais ils ont offert de nombreuses possibilités d'analyse.

\section{Conclusion}

Ce cheminement théorique nous a permis d'envisager différents courants de recherches sur l'expert, avec leurs limites. La première approche étudiée, l'approche cognitive classique, s'est centrée sur un (ou plusieurs) aspect(s) de la cognition, pour tenter d'expliquer l'action en situation standardisée. Il en a résulté une mise à jour des compétences de l'expert, dans son domaine d'expertise. Celui-ci était volontairement réduit, afin de contrôler les différentes variables en jeu. Cette approche a également pu contribuer à élaborer une théorie des tâches. Mais cette voie de recherche n'est pas apparue heuristique pour étudier l'activité de l'expert en situation naturelle.

L'absence ou la faible prise en compte du contexte dans l'approche cognitive classique a été à l'origine d'une controverse. Elle a conduit à concevoir le contexte selon deux angles différents : soit à partir de la notion de situation, soit à partir de l'idée d'un couplage entre l'individu et l'environnement. Dans le premier cas, on s'est intéressé aux composantes liées à la temporalité, au contrôle et à l'incertitude. Dans le second cas, on a insisté sur l'inscription temporelle de la cognition, sur la nature de l'interprétation qui est directement liée au couplage d'un individu à son environnement à un instant, à l'importance de l'intentionnalité qui est à l'origine des significations accordées à la situation et enfin, à l'action. Ces conceptions nuancées du contexte ont amené à une approche particulière de l'homme au travail. Celui-ci n’est plus confronté à des tâches prescrites par une personne extérieure, mais il est engagé dans des situations qui évoluent sans cesse. L’individu n’est plus considéré selon ses compétences identifiées au préalable, mais on le prend en compte dans sa globalité (compétences actualisées, fatigue, émotions, enjeu de la situation pour lui-même et plus largement sens accordé à la situation...) et dans ses interactions avec l'environnement qui évolue avec ou sans lui. Ce couple ne peut plus être dissocié, si l'on veut comprendre l'activité de l'acteur en situation.

En nous appuyant sur une perspective épistémologique inhérente à la cognition, à l'action et au contexte, nous avons envisagé deux approches ergonomiques pour étudier l'activité de l'expert. Si toutes deux ont considéré ce couplage comme étant plus ou moins explicitement inéluctable, leurs postulats et leurs hypothèses ont différé. L'une a été fondamentalement cognitiviste, même si la position était beaucoup plus nuancée que celle des approches " classiques », alors que l'autre a réfuté la notion de représentation. Dans l'une les décisions étaient totalement émergeantes, dans l'autre elles l'étaient partiellement ou totalement, en fonction des circonstances de l'environnement dynamique. L'une a fait l'objet d'études dans le domaine du sport, alors que dans l'autre, les recherches sont balbutiantes. Toutefois, le concept de risque, largement présent dans cette dernière pourrait être mieux considéré dans le domaine du sport. 
Références :

Abernethy, B. (1991). Visual search strategies and decision making in sport. International Journal of Sport Psychology, 22, 189-210.

Abernethy, B. (1993). The nature of expertise in sport. Actes du congrès de l'ISSP, Lisbonne.

Abernethy, B., \& Russel, D.G. (1987). Expert-novice differences in an applied selective attention task. Journal of Experimental Psychology: Learning, Memory and Cognition, 10, 483-495.

Abernethy, B., Wood, J.M., \& Parks, S. (1999). Can the anticipatory skills of experts be learned by novices ? Research Quaterly for Exercice and Sport, 70, 313-318.

Adelson, B. (1984). When novices surpass experts: The difficulty may increase with expertise. Journal of Experimental Psychology, 12, 14-21.

Allard F., Graham, S., \& Paarsalu, M.A. (1980). Perception in sport: Basket-ball. Journal of Sport Psychology, 12, 14-21.

Allard F., \& Starkes J.L. (1980). Perception in sport: volley-ball. Journal of Sport Psychology, 2, 22-33.

Amalberti, R. (1991). Savoirs et savoir-faire de l'opérateur : aspects théoriques et pratiques en ergonomie. In R. Amalberti, M. Montmollin (De), \& J. Theureau (Eds.), Modèles en analyse du travail (pp. 279-294). Liège : Mardaga.

Amalberti, R. (1996). La conduite des systèmes à risques. Paris : P.U.F..

Amalberti, R. (2001). La maîtrise des situations dynamiques. Psychologie Française, 46 (2), 107-118.

Amalberti, R., \& Deblon, F. (1992). Cognitive modelling of fighter aircraft process control: A step towards an intelligent on-board assisance system. International Journal of Man Machine Studies, 36, 639-671.

Amalberti, R., \& Hoc J.M. (1998). Analyse des activités cognitives en situation dynamiques : pour quels buts ? Comment ? Le Travail Humain, 61 (3), 209-234.

Arripe-Longueville (D’), F., Suary, J., Fournier, J., \& Durand, M. (2001). Coach-athlete interaction during elite archery competitions: An application of methodological frameworks used in ergonomics research to sport psychology. Journal of Applied Sport Psychology, 13 (3), 275-299.

Block, K.K., \& Beckett, K.D. (1990). Verbal descriptions of skills by specialists and non specialists. Journal of Teaching in Physical Education, 10, 21-37.

Cellier, J.M. (1996). Exigences et gestion temporelle dans les environnements dynamiques. In J.-M. Cellier, V. De Keyser, \& C. Valot (Eds.), La gestion du temps dans les environnements dynamiques (pp. 19-48). Paris : P.U.F.. 
Chase, W.G., \& Simon, H.A. (1973). Perception in chess. Cognitive Psychology, 4, 55-81.

Chi, M.T.H., Feltovitch, P., \& Glaser, R. (1981). Categorization and representation of physics problems by experts and novices. Cognitive Science, 5, 121-152.

Chiesi, H.L., Spillich, G.J., \& Voss, J.F. (1979). Acquisition of domain related information in relation to high and low domain of knowledge. Journal of Verbal Learning and Verbal Behavior, 18, 257-273.

Christensen, S.A., \& Glencross, D.J. (1993). Expert knowledge and expert perception in sport : Anticipating a field hockey goal shot. Actes du congrès de l'ISSP, Lisbonne.

Clot, Y. (1998). Le travail sans l’homme ? Paris : La Découverte.

Côté, J., Salmela, J.H., \& Russel, S. (1995a). The knowledge of high-performance gymnastic coaches: Methodological framework. The Sport Psychologist, 9, 65-75.

Côté, J., Salmela, J.H., \& Russel, S. (1995b). The knowledge of high-performance gymnastic coaches: Competition and training considerations. The Sport Psychologist, 9, 76-95.

Côté, J., Salmela, J.H. , Trudel, P., Baria, A., \& Russel, S. (1995c). The coaching model: A grounded assesment of expert gymnastic coaches knowledge. Journal of Sport Exercice, $17(1), 1-17$.

Daniellou, F. (1996). Introduction. Questions épistémologiques autour de l'ergonomie. In F. Daniellou (Ed.), L’ergonomie en quête de ses principes (pp. 1-17). Toulouse : Octarès.

Debû, B., Zoudji, B., \& Thon, B. (2003). Mémoire de travail et mémoire implicite : influence de l'expertise en situation de prise de décision en foot-ball. In D. Lehénaff, \& C. Mathieu (Eds.), Expertise et sport de haut niveau, (pp. 229-233). Paris : INSEPPublications.

Donze, B., \& Durand, M. (1997). Etude sur les connaissances pratiques des experts en escalade. Rapport au Ministère de la Jeunesse et des sports (pp. 80-104).

Dosse, F. (1995). L’empire du sens. Paris : La Découverte.

Ericsson, K.A. (1996). The road to excellence. Mahwah: Lawrence Erlbaum associates.

Ericsson, K.A. (2002). The pursuit of excellence in education. Hillsdale NJ: Erlbaum.

Ericsson, K.A., \& Charness, N. (1994). Expert performance: Its structure and acquisition. American Psychologist, 49, 725-745.

Ericsson, K.A., \& Chase, W.G. (1982). Exceptional memory. American Scientist, 70, 607615.

Ericsson, K.A, Chase, W.G., \& Faloon, S. (1980). Acquisition of a memory skill. Science 208, 1181-1182. 
Ericsson, K.A, Krampe, R.T., \& Tesch-Romer, C. (1993). The role of deliberate practice in the acquisition of expert performance. Psychological Review, 100, 363-406.

Ericsson, K.A., \& Lehman, A.C. (1996). Expert and exceptional performance: evidence of maximal adaptation to task constraints. In Annual Review of Psychology, 100, 363-406.

Ericsson, K.A., \& Polson, P.G. (1988). A cognitive analysis of exceptional memory for restaurant orders. In M.T.H. Chi, R. Glaser, \& M.J. Farr (Eds.), The nature of expertise (pp. 23-70). Hillsdale: Lauwrence Erlbaum associates.

Ericsson, K.A., \& Simon, H.A. (1980). Verbal reports as data. Psychological Review, 87, 215251.

Ericsson, K.A., \& Smith, J. (1994). Prospects and limits of the empirical study of expertise: An introduction. In K.A. Ericsson, \& J. Smith (Eds.), Toward a general theory of expertise (pp. 1-38). Cambridge: Cambridge University Press.

Famose, J.P. (1990). Apprentissage moteur et difficulté de la tâche. Paris : INSEP Publications.

Fleishman, E.A., \& Quaintance, M.K. (1984). Taxonomies of human performance. NewYork: Academic Press.

Garfinkel, H. (1967). Studies in ethnomethodology. Englewood Cliffs: Prentice Hall

Garland, D.J., \& Barry, J.R. (1991). Cognitive advantage in sport: The nature of perceptual structures. The American Journal of Psychology, 104, 211-228.

Gibson, J.J. (1979). The ecological approach to visual perception. Boston: Houghton Mifflin co.

Groot (De), A. (1978). Thought and choice in chess. The Hague: Mouton.

Hauw, D., Berthelot, C., \& Durand, M. (2003). Enhancing performance in elite athletes through situated-cognition analysis: Trampolinists' course of action during competition activity. International Journal os Sport Psychology, 34, (4), 299-321

Hollnagel, E. (1993). Human reliability analysis. Context and control. London: Academic Press.

Housner, L.D., Gomez, R., \& Griffey, D.C. (1993). A pathfinder analysis of pedagogical knowledge structures: A follow-up investigation. Research Quaterly for Exercice and Sport 64 (3), 291-299.

Kellog, R .T. (1982). When can we introspect acurately about mental process ? Memory and cognition, 10 (2), 141-144.

Keyser (De), V. (1988). De la contingence à la complexité : l'évolution des idées dans l'étude des processus continus. Le Travail Humain, 51(1), 1-18. 
Lahire, B. (1998). L'homme pluriel. Paris : Nathan.

Lave, J. (1988). Cognition in practice. Cambridge UK: Cambridge University Press.

Leplat, J. (2000). La gestion des communication par le contexte. In B. Melier, \& Y. Queinnec (Eds.), Communication et travail (pp.36-43). Toulouse : Octares.

Leplat J., \& Hoc J.M. (1983). Tâche et activité dans l'analyse psychologique des situations. Cahiers de Psychologie Cognitive, 3 (1), 49-63.

Merleau-Ponty, M. (1945). Phénoménologie de la perception. Paris : Gallimard.

Macquet, A.-C. (2001). Le contrôle des situations chez les volleyeurs experts : informations perçues et connaissances mobilisées ou construites. Thèse de Doctorat non publiée, Université Paris XI.

Miller, G.A. (1956). The magical number seven, plus or minus two. Psychological Review, 63, 81-97.

Montmollin (De), M. (1995). Vocabulaire de l'ergonomie. Toulouse : Octares.

Murphy, G.L., \& Wright, J.C. (1984). Changes in conceptual structure with expertise: Differences between real-world experts and novices. Journal of Experimental Psychology : Learning, Memory and cognition, 10 (1), 144-155.

Newell, A., \& Simon, H.A. (1972). Human problem solving. Englewood Cliffs N.J: Prentice Hall.

Nisbett, R.E., \& Camp Wilson (De), T. (1977). Telling more than you can know: Verbal reports on mental process. Psychological Review, 84 (3), 231-259.

Peirce, C.S. (1978). Ecrits sur le signe. Paris : Seuil.

Raufaste, E. (2001). Les mécanismes cognitifs du diagnostic médical. Paris : P.U.F.

Russel, S.T., \& Salmela, J.H. (1992). Quantifying expert athlete knowledge. Journal of Applied Sport Psychologist, 4, 10-26.

Sanders, A.F. (1986). Energical states underlying task performance. In G. Robert, J. Hockey, A.W.K. Gaillard, \& M.G.H. Coles (Eds.), Energetics and human information processing (pp. 139-154). Dordrecht: Martinus Nijhoff Publishers.

Saury, J., \& Durand, M. (1995). Etude des connaissances pratiques des entraîneurs experts en voile : de l'analyse des relations entraîneurs-athlètes à une modélisation de la situation d’entraînement. Sport, 151, 25-39.

Saury, J., Durand, M., \& Theureau, J. (1997). L'action d'un entraineur expert en voile en situation de compétition : étude de cas. Science et Motricité, 31, 21-35. 
Savelsbergh, G.J.P., Williams, A.M., Van Der Kamp, J., \& Ward, P. (2002). Visual search, anticipation and expertise in soccer goalkeepers. Journal of Sports Sciences, 20, 279287.

Sève, C., \& Durand (1999). L'action de l'entraîneur comme action située. Avante,5, 1, 69-86.

Seve, C., Saury, J. Theureau, J., \& Durand, M. (2002). La construction de connaissances chez les sportifs de haut niveau lors d'une interaction compétitive. Le Travail Humain, 65 (2), 159-190.

Simon, H.A. (1973). The structure of ill-structured problems. Artificial Intelligence, 4, 181201.

Sperber, D. (1996). La contagion des idées. Paris : Odile Jacob.

Starkes, J.L. (1987). Skill in field hockey: The nature of cognitive advantage. Journal of Sport Psychology, 9 (2), 146-159.

Strauss, A., \& Corbin, J. (1990). Basics of qualitative research: Grounded theory procedures and techniques. Berverly Hills, CA: Sage.

Suchman, L.A. (1987). Plans and situated actions. Cambridge: Cambridge University Press.

Temprado, J.J., \& Famose, J.P. (1993). Analyse de la difficulté informationnelle et description des tâches motrices. In J.P. Famose (Ed.), Cognition et performance (pp. 165-182). Paris : INSEP Publications.

Theureau, J. (1992). Le cours d'action : analyse sémiologique. Berne : Peter Lang.

Theureau, J., \& Jeffroy, F. (1994). Ergonomie des situations informatisées. Toulouse: Octares.

Valot, C. (1996). Gestion du temps. Gestion du risque. In J.M. Cellier, V. De Keyser, \& C. Valot (Eds.), La gestion du temps dans les environnements dynamiques (pp. 244-265). Paris : P.U.F..

Van Daele, A. (1997). Contribution de la simulation à l'étude de l'activité de l'opérateur en situation dynamique. In P. Beguin, \& A. Weill-Fassina (Eds.), La simulation en ergonomie : connaître, agir et interagir (pp. 29-37). Toulouse : Octares.

Van Daele, A, \& Carpinelli, F. (1997). Anticipation de l'action et anticipation du processus : l'influence de la situation. In J.M. Cellier, V. De Keyser, \& C. Valot (Eds), La gestion du temps dans les environnements dynamiques (pp. 200-220). Paris : P.U.F..

Varela, F.J. (1989a).Connaître les sciences cognitives. Paris : Seuil.

Varela, F.J. (1989b). Autonomie et connaissance. Paris : Seuil.

Varela, F.J. ; Thompson, E. ; Rosch, E. (1993). L'inscription corporelle de l'esprit. Paris : Seuil. 
Williams, M. (2003). L'évaluation des comportements de recherche visuelle en sport. In D. Lehénaff, \& C. Mathieu (Eds), Expertise et sport de haut niveau (pp. 125-127). Paris : INSEP-Publications.

Williams, M., \& Davids, N. (1995). Declarative Knowledge in sport: A by-product of experience or a characteristic of expertise ? Journal of Sport and Exercice Psychology, $17,259-275$.

Williams, M., \& Elliott, D. (1999). Anxiety and visual search in karate. Journal of Sport and Exercice Psychology, 21 (4), 362-375.

Wisner, A. (1996). Questions épistémologiques en ergonomie et en analyse du travail. In F. Daniellou (Ed.), L'ergonomie en quête de ses principes (pp. 29-55). Toulouse : Octares.

Wright, D.L., Pleasant, F., \& Gomez-Meza, M. (1990). Use of advance visual sources in volley-ball. Journal of Sport and Exercice Psychology, 12, 406-414. 
Tableau 1

Les Etudes liées aux Compétences Perceptives des Experts en Référence à l' Approche Cognitive Computationnelle

\section{Chase \& Simon (1973)}

- Echecs

- But : connaître les perceptions de l'expert.

Cette étude a fait partie des

" études pionnières ».

Chiesi, Spillich \& Voss (1979)

- Base-ball

- But : envisager l'influence des

connaissances acquises dans un

domaine sur l'acquisition de

connaissances dans un autre

domaine.

Allard, Graham, \& Paarsalu

(1980)

- Basket-ball

- But : étudier la relations entre

performance et perception en

sport.

Cette étude s'est appuyée sur les

travaux de Chase et Simon (1973)

Allard \& Starkes (1980)

- Volley-ball

- But : comparer la vitesse et la

précision de la détection du ballon sur diapositives.

$\begin{array}{ll}\text { 1. Perception : reproduire le } & 3 \text { groupes : } \\ \text { placement des pièces sur un } & \text { - experts (maîtres d'échec) } \\ \text { autre échiquier. } & \text { - joueurs de niveau intermédiaire } \\ \begin{array}{l}\text { 2. Mémoire : observation des } \\ \text { pièces pendant } 5 \text { sec et }\end{array} & \text { novices }\end{array}$

pièces pendant 5 sec et

placement des pièces.

Tâche de rappel : écouter une série de situations de jeu et indiquer si elles sont nouvelles ou si elles ont fait

l'objet d'une présentation préalable.

2 groupes : sujets présentant un haut (HK) ou bas niveau de connaissances (LK) en base-ball

(évaluation à partir d'un questionnaire)
1. Les experts étaient plus

rapides, leurs configurations ne

semblaient pas être rapportées au

hasard.

2. La taille des « chunks » était

plus grande chez les experts.
Les experts percevaient plus

d'informations que les autres

sujets lors d'une brève

observation.

Les pièces seraient reliées entre elles à partir de relations abstraites.

Les HK détectaient mieux les

changements de la structure du jeu que les LK.

Les experts accédaient plus

rapidement aux connaissances contenues en MLT. n domaine pouvaient faciliter l'acquisition d'informations dans un nouveau domaine lié au précédent.

$\begin{array}{ll}\text { Tâche de rappel : } 20 & 2 \text { groupes : } \\ \text { diapositives présentant du jeu } & \text { - un groupe de basketteuses de N1 }\end{array}$

1. Les joueurs rappelaient plus

d'informations en condition de

jeu structuré

structuré ou non.

- un groupe de non basketteuses

Tâche de reconnaissance :

xperts traitaient plus

indiquer si la situation a déjà

deinformations sur le jeu

structuré.

Les résultats étaient consistants avec ceux obtenus aux échecs.

Les experts avaient des

performances supérieures en

condition de jeu structuré (/ non experts).

\section{Tâche de détection : \\ projection de diapos de jeu \\ 2 groupes: \\ - équipe première de l'université}

structuré ou non, durant 16,65 - non joueurs

Indiquer le placement du

ballon

(vitesse et précision requises).
Il n’y a pas eu de différence dans Les résultats étaient consistants la précision des réponses entre les groupes et les conditions. Les experts étaient plus rapides que les non joueurs. avec ceux obtenus au basket-

ball : rappel supérieur chez les experts en condition de jeu structuré. 


\begin{tabular}{|c|c|c|c|c|}
\hline $\begin{array}{l}\text { Starkes (1987) } \\
\text { - Hockey sur gazon } \\
\text { - But : étudier des habiletés } \\
\text { cognitives ou des attributs } \\
\text { « software » d'un groupe de } \\
\text { hockeyeurs experts. }\end{array}$ & $\begin{array}{l}\text { 1. Tâche d'acuité visuelle } \\
\text { dynamique } \\
\text { 2. Tâche de rappel }\end{array}$ & $\begin{array}{l}3 \text { groupes : } \\
\text { - équipe nationale } \\
\text { - équipe de première division } \\
\text { (varsity) } \\
\text { - novices }\end{array}$ & $\begin{array}{l}\text { 1. Il n’y avait pas de différence } \\
\text { entre les groupes dans la } \\
\text { poursuite de l'objet en } \\
\text { mouvement } \\
\text { 2. Le rappel en jeu structuré était } \\
\text { meilleur pour l'équipe nationale. }\end{array}$ & $\begin{array}{l}\text { Les décisions des experts } \\
\text { n’étaient pas plus rapides que } \\
\text { celles des autres groupes, mais } \\
\text { elles étaient plus précises. }\end{array}$ \\
\hline $\begin{array}{l}\text { Abernethy \& Russel (1987) } \\
\text { - Badminton } \\
\text { - But : comparer les données } \\
\text { spatiales et temporelles utilisées } \\
\text { par les experts et les novices pour } \\
\text { anticiper les actions. }\end{array}$ & $\begin{array}{l}\text { Tâche d'anticipation : 1. en } \\
\text { condition d'occlusion } \\
\text { temporelle de la frappe du } \\
\text { volant, évaluer la trajectoire } \\
\text { après la frappe } \\
\text { 2. idem en condition } \\
\text { d'occlusion spatiale }\end{array}$ & $\begin{array}{l}2 \text { groupes } \\
\text { - experts (participation aux Jeux du } \\
\text { Commonwealth) } \\
\text { - novices (joueurs non réguliers) }\end{array}$ & $\begin{array}{l}\text { Les experts observaient le bras } \\
\text { par rapport à la raquette, les } \\
\text { novices se centraient moins sur } \\
\text { ces parties. } \\
\text { Les experts extrayaient plus tôt } \\
\text { l'information, que les novices. }\end{array}$ & $\begin{array}{l}\text { Les performances dans la tâche } \\
\text { étaient liées au niveau des } \\
\text { joueurs. } \\
\text { Les experts utilisaient davantage } \\
\text { les informations proximales que } \\
\text { les novices. }\end{array}$ \\
\hline $\begin{array}{l}\text { Wright, Pleasant \& Gomez-Meza, } \\
\text { (1990) } \\
\text { - Volley-ball } \\
\text { - But : étudier la capacité à } \\
\text { anticiper les données visuelles. }\end{array}$ & $\begin{array}{l}\text { Tâche d'anticipation en } \\
\text { condition d'occlusion } \\
\text { temporelle : identifier le lieu } \\
\text { de frappe (par rapport à la } \\
\text { ligne d'attaque) et préciser les } \\
\text { informations perçues pour } \\
\text { prendre la décision }\end{array}$ & $\begin{array}{l}2 \text { groupes : } \\
\text { - joueurs de } 1^{\text {ère }} \text { division nationale } \\
\text { - étudiants non diplômés }\end{array}$ & $\begin{array}{l}\text { La période temporelle de } 167 \\
\text { msec avant l'impact est apparue } \\
\text { importante. } \\
\text { Les « experts » prenaient des } \\
\text { informations plus pertinentes sur } \\
\text { le jeu (position du corps, élan de } \\
\text { l'attaquant). }\end{array}$ & $\begin{array}{l}\text { L’habileté d’anticipation était } \\
\text { supérieure chez les joueurs } \\
\text { expérimentés }\end{array}$ \\
\hline $\begin{array}{l}\text { Abernethy (1991) } \\
\text { - Badminton et squash } \\
\text { - Buts : identifier la nature des } \\
\text { informations précoces et } \\
\text { l'utilisation de ces informations } \\
\text { en relation avec le niveau } \\
\text { d'expertise des joueurs. }\end{array}$ & $\begin{array}{l}\text { Tâche d'anticipation en } \\
\text { condition d'occlusion spatiale } \\
\text { et temporelle } \\
\text { A partir d'une épreuve } \\
\text { simulée au cinéma, le sujet } \\
\text { doit prédire un service }\end{array}$ & $\begin{array}{l}2 \text { groupes : } \\
\text { - experts } \\
\text { - novices }\end{array}$ & $\begin{array}{l}\text { Le mouvement du bras et de la } \\
\text { raquette ont constitué des } \\
\text { informations critiques précoces. } \\
\text { On a observé une relation entre } \\
\text { le comportement visuel et le } \\
\text { déroulement proximo-distal du } \\
\text { service. }\end{array}$ & $\begin{array}{l}\text { Les experts prévoyaient mieux la } \\
\text { trajectoire (vitesse et direction) à } \\
\text { partir d'informations précoces. }\end{array}$ \\
\hline $\begin{array}{l}\text { Garland \& Barry (1991) } \\
\text { - Football } \\
\text { - But : étudier la nature des } \\
\text { processus cognitifs utilisés par } \\
\text { l'expert. }\end{array}$ & $\begin{array}{l}\text { 1. Tâche de rappel avec } 5 \text { sec } \\
\text { d'observations de la position } \\
\text { des joueurs } \\
\text { 2. Tâche de reconnaissance et } \\
\text { de rassemblement : } \\
\text { classification d'images liées } \\
\text { au football }\end{array}$ & $\begin{array}{l}2 \text { groupes : } \\
\text { - Experts ( } 5 \text { ans de pratique) } \\
\text { - Non experts ( } 2 \text { ans de pratique) }\end{array}$ & $\begin{array}{l}\text { 1. Les experts rappelaient des } \\
\text { configurations de jeu plus larges, } \\
\text { après } 5 \text { sec d'observation, que les } \\
\text { novices (hypothèse de } \\
\text { regroupement conceptuel). } \\
\text { 2. Leur structure d'organisation } \\
\text { était plus discriminative et plus } \\
\text { rapide. }\end{array}$ & $\begin{array}{l}\text { L’hypothèse du regroupement } \\
\text { conceptuel a été proposé comme } \\
\text { alternative à l'hypothèse du } \\
\text { regroupement perceptif. } \\
\text { Les résultats étaient consistants } \\
\text { avec ceux obtenus aux échecs (la } \\
\text { structure d'organisation en MLT } \\
\text { facilitait la mémorisation). }\end{array}$ \\
\hline
\end{tabular}


Williams \& Elliott (1999)

- Karaté

- Buts : étudier des effets de

l'anxiété sur les stratégies de

recherche visuelle.

Tâche d'anticipation et de

recherche visuelle : esquive des

2 groupes :

mouvements adverses

représentés sur écran

Conditions : anxiété ou absence karaté

d'anxiété

Mesure : vitesse et précision du

rappel

Savelsbergh, Williams, Van der

Kamp \&Ward (2002)

- Fotball

Tâche d'anticipation : à partir

d'un film, anticiper la direction

du penalty

- Buts : étudier les stratégies de

Mesures : vitesse et précision du

recherche visuelle des gardiens.

rappel
- experts (14,3 ans de pratique

compétitive en moyenne)

- novices pas d'entraînement en

1. L’anxiété avait des effets sur

2 groupes : Le côté du tir était mieux prévu

- experts (2è division nationale) que la hauteur, pour les deux

Les performances d'anticipation

- novices (jeu récréatif) les focalisations du regard.

2. En condition d'anxiété, les

novices diminuaient leur durée

de fixation visuelle ; les experts

utilisaient des temps plus longs,

leur habileté perceptive était

supérieure (vitesse et précision

des réponses).

groupes.

Les experts étaient plus précis

étaient meilleures chez les

experts. Ils prenaient moins

d'informations que les novices.

d’un rétrécissement périphérique

qui a incité le sujet à prendre

plus d'informations sur le

en vision centrale,

qu’en vision périphérique.

sur la hauteur du tir. 
Tableau 2

Les Etudes liées aux Compétences Mnésiques des Experts en Référence à l'Approche Cognitive Computationnelle

\begin{tabular}{|c|c|c|c|c|}
\hline Auteurs et études & Protocoles & Sujets & Résultats & Synthèse \\
\hline $\begin{array}{l}\text { Ericsson, Chase \& Faloon (1980) } \\
\text { - Signes } \\
\text { - But : proposer un modèle dans } \\
\text { lequel les progrès des sujets au } \\
\text { niveau de la capacité de la } \\
\text { mémoire à court terme (MCT) } \\
\text { seraient liés à une utilisation plus } \\
\text { efficace de la mémoire à long } \\
\text { terme (MLT). }\end{array}$ & $\begin{array}{l}\text { Tâche de mémorisation de } \\
\text { signes, à partir d’une pratique } \\
\text { intense ( } 3 \text { à } 5 \text { heures } \\
\text { hebdomadaires pendant plus de } \\
20 \text { mois) } \\
\text { Mesure : quantité d'items } \\
\text { rappelés avec précision }\end{array}$ & étudiant & $\begin{array}{l}\text { Son empan mnésique a } \\
\text { augmenté : passage de } 7 \text { (mesure } \\
\text { de Miller, 1956) à plus de } 80 \\
\text { items. } \\
\text { Sa capacité à rappeler les items } \\
\text { après une session a également } \\
\text { augmenté. }\end{array}$ & $\begin{array}{l}\text { L’habileté de mémoire était } \\
\text { largement liée à l'association } \\
\text { d'un matériau non connu avec un } \\
\text { matériau familier. Ceci a permis } \\
\text { de soulager la MCT dans la } \\
\text { mesure où le rappel pouvait être } \\
\text { réalisé à partir d'une simple } \\
\text { association dans un code déjà } \\
\text { existant en MLT. }\end{array}$ \\
\hline $\begin{array}{l}\text { Ericsson \& Polson (1988) } \\
\text { - Restauration } \\
\text { - But : élaborer un modèle lié à } \\
\text { l’habileté mnémonique. }\end{array}$ & $\begin{array}{l}\text { Tâche de rappel : association } \\
\text { d'une commande de plats avec } \\
\text { un visage et rappel } \\
\text { Mesure : quantité de réponses } \\
\text { rappelées avec précision }\end{array}$ & $\begin{array}{l}2 \text { groupes : } \\
\text { - un serveur expérimenté } \\
\text { - des sujets non entraînés } \\
\text { (étudiants) }\end{array}$ & $\begin{array}{l}\text { Le serveur codait les items par } \\
\text { groupes de tables. } \\
\text { Les étudiants réalisaient un } \\
\text { rappel ordonné de listes. } \\
\text { Les performances du serveur } \\
\text { étaient plus élevées. }\end{array}$ & $\begin{array}{l}\text { Les procédures du serveur étaient } \\
\text { flexibles. } \\
\text { Il accédait plus facilement à ses } \\
\text { structures mnémoniques. }\end{array}$ \\
\hline $\begin{array}{l}\text { Debû, Zoudji \& Thon (2003) } \\
\text { - Football } \\
\text { - But : envisager l’implication de } \\
\text { la MLT dans les phénomènes } \\
\text { d’amorçage par répétition, tester } \\
\text { la mémoire implicite chez les } \\
\text { footballeurs experts. }\end{array}$ & $\begin{array}{l}\text { Tâche de prise de décision : dans } \\
\text { une situation de jeu particulière, } \\
\text { qui pouvait être présentée deux } \\
\text { fois, à l'insu des sujets. La } \\
\text { vitesse de choix était requise } \\
\text { Mesure : quantité de réponses } \\
\text { rapides et précises }\end{array}$ & $\begin{array}{l}2 \text { groupes : } \\
\text { - footballeurs professionnels } \\
\text { (plus de } 12 \text { ans de pratique) } \\
\text { - novices (pas de pratiques } \\
\text { compétitive et peu d'intérêt pour } \\
\text { le foot) }\end{array}$ & $\begin{array}{l}\text { Les réponses des experts étaient } \\
\text { supérieures à celles des novices. } \\
\text { Le TR des experts était toujours } \\
\text { plus faible que celui des novices. } \\
\text { On a observé un effet } \\
\text { d'amorçage lors de la seconde } \\
\text { présentation. }\end{array}$ & $\begin{array}{l}\text { Les mécanismes de } \\
\text { mémorisation implicite étaient } \\
\text { indépendants de la charge de la } \\
\text { mémoire de travail. } \\
\text { Le fonctionnement des deux } \\
\text { groupes a été identique, avec une } \\
\text { efficacité accrue des experts. }\end{array}$ \\
\hline
\end{tabular}


Tableau 3

Les Etudes liées au Raisonnement et à l'Evaluation des Bases de Connaissances des experts, en référence à l'Approche Cognitive Computationnelle

\section{Auteurs et études}

De Groot (1978)

- Echecs

- But : identifier les différences experts/novices et élaborer une série de tâches qui évalue leur niveau d'expertise.

\section{Chi, Feltovitch \& Glaser (1981)}

- Physique

- But : comprendre les

représentations mentales des

individus.

\section{Murphy \& Wright (1984)}

- Troubles psychologiques chez

l'enfant

- But : étudier les différences

structurales entre experts et

novices au niveau des concepts

liés aux troubles psychologiques chez l'enfant.

\section{Adelson (1984)}

- Informatique

- But : étudier les représentations

liées aux programmations

informatiques entre experts et

novices.

\section{Block \& Beckett (1990)}

- Lancer athlétique

- But : étudier la précision de la

\begin{tabular}{ll}
\multicolumn{1}{c}{ Protocoles } & \multicolumn{1}{c}{ Sujets } \\
Protocole de pensée à voix & 3 groupes : \\
haute : les joueurs devaient & - grands maîtres \\
décider de la meilleure action à & - experts \\
réaliser dans des configurations & - novices \\
de jeu différentes & \\
Mesures : quantité de réponses & \\
efficaces, temps de réponse &
\end{tabular}

\section{2 groupes}

- experts

raisonnement : les sujets

devaient classer et conceptualiser - novices

les problèmes pour les résoudre

Mesures : temps de réponse et

mode de classification des

problèmes

\section{Tâche de conceptualisation : les 4 groupes :}

sujets devaient décrire les

caractéristiques d'un enfant

agressif, déprimé, et qui

présentait des troubles du

comportement.

Mesures : quantité et nature des attributs rapportés par catégories

Protocole verbal : les sujets devaient expliquer le

onctionnement et la nature des tâches exécutées par le programme informatique

Mesures : contenu des

représentations (nature et nombre d'informations)

$\begin{array}{ll}\text { Protocole verbal : description } & 2 \text { groupes d'enseignants en cycle } \\ \text { précise d'une habileté de lancer } & \text { élémentaire : }\end{array}$

-experts (superviseurs du

programme d'enseignement)

- conseillers experts (étudiants

du programme d'été)

- conseillers débutants (novices)

- étudiants dans un domaine autre que celui de la psychologie.

\section{2 groupes :}

- experts (enseignant en

programmation informatique)

- novices (étudiants en

programmation informatique) de balle - spécialistes (4 ans d'études

\begin{tabular}{l}
\multicolumn{1}{c}{ Résultats } \\
Les joueurs expérimentés \\
trouvaient des solutions plus \\
efficaces que les autres. \\
Les grands maîtres prenaient 10 \\
min avant de déplacer les pièces. \\
Ils envisageaient une trentaine de \\
possibilités de jeu pour chaque \\
configuration.
\end{tabular}
configuration.

Les novices ne s'intéressaient

qu'aux traits de surface

apparaissant dans l'énoncé.

Les experts traitaient rapidement

l'information en fonction de la

solution du problème.

\section{Synthèse}

Le choix du mouvement pour une position des pièces a permis de mettre à jour les processus cognitifs qui différenciaient les joueurs.

Les experts classaient les problèmes en fonction

d'associations signifiantes (leurs catégories reflétaient les principes physiques sous-jacents aux solutions).

Les experts rapportaient plus

La précision de la description

d'attributs que les novices pour

chacune des catégories. Les traits

qu'ils rapportaient étaient plus globaux.

était liée au niveau d'expertise.

Les représentations des experts contenaient des informations Les représentations des experts générales sur « ce que faisait » le d'actions) alors que celles des programme. novices étaient concrètes Celles des novices renfermaient (fonctionnement). des informations sur le fonctionnement du programme.

Les spécialistes posaient

Les descriptions des spécialistes davantage de questions sur la étaient plus longues et plus tâche (utile à l'habileté) que les

précises que celles des non 
description de l'habileté de lancer par des éducateurs

spécialistes ou non.
Mesures : contenu des

verbalisations (nature et $\mathrm{qua}$

de verbalisations liées aux

différentes catégories rapportées) supplémentaires par rapport au non spécialistes.

groupe non spécialiste)

- non spécialistes connaissances de l'athlète et ses

\section{Russel \& Salmela (1992)}

- Cyclisme

- But : mettre à jour les

structures de connaissances.
1.Entretien structuré visant à connaître les problèmes sportifs ou les tâches perçues comme

\section{Un homme, de niveau}

consistantes

2.Classement de situations

représentées sur des cartes

le protocole était basé sur la

théorie ancrée

Mesures : contenu des

verbalisations (nature et

quantités des connaissances

rapportées)

Housner, Gomez \& Griffey

(1993)

- Pédagogie

- But : étudier la validité externe

de bases de connaissances

pédagogiques et comparer les

étudiants avec cinq programmes

d'éducation.
Tâche : à l'issue de cours de méthodologie, examen de

connaissances portant sur une

liste de concepts

Mesures : contenu des

connaissances des sujets (nombre

et structuration) structures de connaissances des international depuis plus de 13

ans
1. Dix-neuf catégories ont été répertoriées et utilisées pour la seconde étude.

2. Six stratégies ont été utilisées pour classer les 19 catégories.

On n’a pas su pourquoi il utilisait certaines stratégies par rapport à d'autres.

2 groupes :
- formateurs enseignants (pour
l'ensemble des cours)
- étudiants en formation (issus de
différentes classes)

Les connaissances des

enseignants étaient plus

consistantes que celles des

étudiants.

Les connaissances des étudiants

augmentaient avec les cours.

Leurs structures de

connaissances se rapprochaient

progressivement de celles des formateurs.

Christensen \& Glencross (1993) 1.Entretien semi-structuré afin de 1. 2 groupes :

- Hockey sur gazon

mettre à jour les informations

- entraîneurs professionnels

- But : étudier les connaissances utilisées par le gardien pendant le nationaux

et les structures de connaissances penalty.

utilisées par les gardiens de

2.Questionnaire : les sujets

- gardiens expérimentés (6 ans de 2

pratique en Nationale 1$)$

hockey. devaient préciser s’ils

2. 3 groupes mixtes :

- experts

comme importantes ou non (s’ils - niveau intermédiaire

les utilisaient ou non).

- novices
1.Les résultats ont permis de mettre à jour des connaissances déclaratives et procédurales.

2.Il n’y avait pas de différences

dans le genre.

Les experts et les sujets

intermédiaires utilisaient moins

d'informations que les novices. spécialistes.

Les catégories et les stratégies

sont apparues comme des

connaissances opérationnelles du

cycliste. Elles ont indiqué

l'organisation cognitive des

connaissances du sujet.

Les catégories ont fourni un profil des types de situations auxquelles le sujet devait faire face.

La structure de connaissances a augmenté avec la formation et sa cohérence est devenue plus forte avec celle des formateurs.

Les sujets n’ont pas utilisé les mêmes informations, selon leur niveau.

On a considéré que les sujets experts et intermédiaires utilisaient des informations plus pertinentes que les novices.

Mesures : quantité et nature des

connaissances rapportées 
- Football base de connaissance et niveau d'expertise et envisager une relation entre anticipation et reconnaissance. basée sur un film présentant des situations de jeu structuré ou non 2. Tâche de rappel : fondée sur des séquences du film utilisé dans la tâche précédente

3.Test de reconnaissance Mesures : de la vitesse et de la précision des réponses

\begin{abstract}
Côté, Salmela \& Russel (1995a)
- Entraîneurs en gymnastique

- Buts : introduire une série

d'articles liés à l'expertise des athlètes et fournir un cadre général de la méthodologie utilisée.
\end{abstract}

\begin{tabular}{ll}
\hline Côté, Salmela \& Russel (1995b) & Entretien semi-directif mené à \\
- Entraîneurs en gymnastique & partir d'un guide \\
- But : mettre à jour les & Mesures : nature et quantité de \\
connaissances des entraîneurs & connaissances rapportées par \\
experts dans la compétition. & catégories \\
& $\begin{array}{l}\text { Le protocole était basé sur la } \\
\text { théorie ancrée (théorie qui part }\end{array}$
\end{tabular}

Entretien semi-directif (guide) Elaboration du cadre théorique de la méthode qui s'appuyait sur la théorie ancrée (méthode qualitative pour collecter et analyser les données de façon inductive (Strauss \& Corbin,

partir d'un guide catégories des faits)
17 entraîneurs experts répondant à trois critères : 10 ans d'expérience minimum, international et de deux gymnastes nationaux minimum, reconnaissances de leurs compétences par la fédération.

1 groupe composé des 17 entraîneurs de l'étude précédente formation d'un gymnaste catégorisées en 134 propriétés.

Côté, Salmela, Trudel, Baria \& Russel (1995c)

- Entraîneurs en gymnastique - But : identifier et conceptualiser les connaissances des entraîneurs en gymnastique.
Entretiens semi-directif menés à partir d'un guide Mesures : nature et quantité de connaissances rapportées par catégories
1 groupe : entraîneurs hommes et femmes experts (10 ans d'expérience minimum formation d'un gymnaste international et de deux gymnastes nationaux minimum meilleures performances

d'anticipation.

2. Le rappel était supérieur pour les experts dans les conditions de jeu structuré.

3. Les experts utilisaient leurs connaissances pour mieux traiter et stocker les informations en mémoire à long terme.

Les connaissances déclaratives étaient procéduralisées plus efficacement à travers la performance (par rapport à l'observation).

Les 17 retranscriptions ont été divisées en 1155 unités significatives, qui ont été et rassemblées dans des catégories plus larges, en fonction de leurs composants.

\section{Deux composantes ont été mises \\ Les connaissances des}

à jour : la compétition (records, performances) et l'entraînement (implication de l'entraîneur, style d'intervention, habiletés techniques, mentales).

Les entraîneurs s'impliquaient peu le jour de la compétition, sauf lorsque les gymnastes n’obtenaient pas les performances escomptées.

étaient plus élaborées et plus spécifiques chez les experts par rapport aux non experts et aux spectateurs (les BC des non experts étaient supérieures à celles des spectateurs). Il a semblé qu'une petite quantité de pratique facilitait le développement des connaissances déclaratives spécifiques à la tâche.

Le rôle des connaissances tacites est apparu important.

Cette approche qualitative s'est fondée sur une méthode de comparaison constante des données, jusqu'à ce qu'elles soient saturées. entraîneurs experts ont fourni des explications détaillées sur les

aspects importants liés au développement de l'élite.

entuaient leurs aractéristiques personnelles, celles des athlètes, leur niveau de connaissances liées à l'athlète, à développement, les facteurs l'entraîneur, à la compétition et à contextuels, pour pouvoir l'entraînement. estimer le potentiel du gymnaste. Il a semblé peu probable que les 
Ces travaux ont constitué des

études princeps inhérentes aux

Le protocole était basé sur la théorie ancrée.

reconnaissances de leurs compétences par la fédération.
Cette évaluation était utilisée pour définir les connaissances

mportantes et les composantes

liées à la compétition, à entraîneurs aient eu un schéma

tout prêt qui contenait les

connaissances génériques

nécessaires au développement de

entraîneurs (en tant qu'acteur

l'organisation et à l'entraînement l'athlète. 
Tableau 4

Les Etudes Liées à l'Activité des Experts en Référence à l'Approche de l'Anthropologie Cognitive Située

\begin{tabular}{|c|c|c|c|c|}
\hline Auteurs et études & Protocoles & Suje & Résultats & Synthèse \\
\hline $\begin{array}{l}\text { - Saury \& Durand (1997) } \\
\text { - Voile } \\
\text { - Buts : analyser l'expertise et } \\
\text { favoriser la conception d'aides à } \\
\text { la formation des entraîneurs. }\end{array}$ & $\begin{array}{l}\text { - Situations naturelles extraites } \\
\text { de } 5 \text { périodes de préparation de } \\
\text { régate (avant, pendant, entre } \\
\text { deux régates) } \\
\text { - } 2 \text { types de recueil de données : } \\
\text {. données d'observation et } \\
\text { d'enregistrement pendant les } \\
\text { compétition } \\
\text {. données de verbalisations } \\
\text { provoquées lors d'entretiens a } \\
\text { posteriori (50 min), menés } 2 \text { à } 5 \\
\text { heures après la compétition } \\
\text { - traitement des données selon le } \\
\text { cadre de l'analyse sémio-logique } \\
\text { du cours d'action }\end{array}$ & $\begin{array}{l}3 \text { athlètes de l'équipe de France } \\
\text { Olympique de voile et leur } \\
\text { entraîneur qui travaillaient } \\
\text { ensemble depuis plus de } 8 \text { ans : } \\
\text { - entraîneur : } 20 \text { ans d'expérience } \\
\text { d'entraînement dont } 10 \text { dans le } \\
\text { cadre de la préparation } \\
\text { olympique } \\
\text { - athlètes : participation à leur } \\
\text { deuxième préparation olympique, } \\
3 \text { podiums aux championnats du } \\
\text { monde depuis } 1992\end{array}$ & $\begin{array}{l}\text { Les actions de l'entraîneur se } \\
\text { structuraient en } 4 \text { points : } \\
\text { - organisation de l'action en } \\
\text { fonction de la distinction } \\
\text { " compétition d'entraînement » } \\
\text { et « ompétition de performance » } \\
\text { - organisation de l'action en } \\
\text { fonction de la distinction « avant } \\
\text { la } 1^{\text {ère }} \text { régate du jour » et « entre } \\
2 \text { régates ». } \\
\text { - engagement de l'entraîneur en } \\
\text { parallèle dans des histoires } \\
\text { différentes avec chaque athlète } \\
\text { - enchaînement de séquences } \\
\text { (engager la communication, faire } \\
\text { expliciter la régate...). }\end{array}$ & $\begin{array}{l}\text { L'action était organisée de façon } \\
\text { intrinsèque à partir de domaines } \\
\text { d'actions significatifs pour } \\
\text { l'entraîneur (type de compétition, } \\
\text { instant). Ces domaines ont été à } \\
\text { l'origine d'une activité } \\
\text { d'interprétation qui a orienté } \\
\text { l'action proprement dite. } \\
\text { L’action de l'entraîneur était } \\
\text { située, elle se construisait } \\
\text { progressivement (prise en } \\
\text { compte du contexte). } \\
\text { Elle était intégrée dans des } \\
\text { systèmes coopératifs. } \\
\text { Ces actions s'inscrivaient dans } \\
\text { des temporalités différentes. }\end{array}$ \\
\hline $\begin{array}{l}\text { - Donzé \& Durand (1997) } \\
\text { - Escalade } \\
\text { - Buts : analyser les } \\
\text { compétences partagées de } \\
\text { différents experts } \\
\text { - } 3 \text { axes de recherche : la nature } \\
\text { des tâches réalisées par les } \\
\text { grimpeurs, l'activité cognitive } \\
\text { mobilisée pour réaliser ces } \\
\text { tâches et la nature des } \\
\text { connaissances utilisées dans } \\
\text { l'action }\end{array}$ & $\begin{array}{l}\text { - } 5 \text { séances d'entraînement, une } \\
\text { ouverture de voie et une } \\
\text { compétition } \\
\text { - } 2 \text { types de recueil de données : } \\
\text {. données d'observation et } \\
\text { d'enregistrement pendant les } \\
\text { entraînements } \\
\text {. données de verbalisation lors } \\
\text { d'entretiens approfondis menés } \\
\text { immédiatement après les } \\
\text { situations pratiques observées, au } \\
\text { pied du mur } \\
\text { - traitement des données selon le } \\
\text { cadre de l'analyse sémio-logique } \\
\text { du cours d'action }\end{array}$ & $\begin{array}{l}\text { - } 3 \text { types de participants : } \\
\text {. } 3 \text { entraîneurs nationaux ayant } \\
\text { un vécu du haut niveau en tant } \\
\text { qu'athlète } \\
\text {. } 3 \text { ouvreurs de voie en } \\
\text { compétition internationale, dont } \\
\text { les compétences étaient } \\
\text { reconnues par leurs pairs depuis } \\
\text { plus de } 10 \text { ans } \\
.5 \text { grimpeurs de l'équipe de } \\
\text { France, classés parmi les } 10 \\
\text { meilleurs mondiaux }\end{array}$ & $\begin{array}{l}\text { Les grimpeurs avaient des } \\
\text { planifications d'entraînement qui } \\
\text { étaient largement diffuses et qui } \\
\text { se précisaient en fonction des } \\
\text { voies à grimper et de leur état } \\
\text { physique et mental. } \\
\text { La construction des tâches } \\
\text { s'établissait à partir de } \\
\text { l'organisation spatiale des prises } \\
\text { (création de parcours ou } \\
\text { mémorisation des mouvements } \\
\text { réalisés). } \\
\text { La prise en compte des émotions } \\
\text { contribuait à organiser l'action } \\
\text { d'entraînement. }\end{array}$ & $\begin{array}{l}\text { Le grimpeur cherchait en } \\
\text { permanence des solutions } \\
\text { optimales. } \\
\text { Les planifications devaient être } \\
\text { souples, elles servaient de } \\
\text { ressources pour l'action située. } \\
\text { Les planifications prenaient } \\
\text { davantage en compte des } \\
\text { sensations physiques et mentales, } \\
\text { qu'une organisation temporelle } \\
\text { de l'action. } \\
\text { Le grimpeur était couplé à } \\
\text { l'environnement, il « fusionnait » } \\
\text { avec lui au cours de l'action. }\end{array}$ \\
\hline $\begin{array}{l}\text { - Sève \& Durand (1999) } \\
\text { - Tennis de table }\end{array}$ & $\begin{array}{l}\text { - } 4 \text { séances d’une durée comprise } \\
\text { entre } 2 \text { et } 3 \text { heures }\end{array}$ & $\begin{array}{l}\text { - } 3 \text { entraîneurs nationaux } \\
\text { masculins, responsables d'une }\end{array}$ & $\begin{array}{l}\text { Ils ont déterminé : } \\
\text { - les séries constitutives de }\end{array}$ & $\begin{array}{l}\text { L'action présentait un caractère } \\
\text { situé, dans un souci }\end{array}$ \\
\hline
\end{tabular}


- But : analyser les composantes temporelles de l'action des entraîneurs experts au cours de séances d'entraînement.
- 2 types de recueil de données : . données d'observation et

d'enregistrement pendant les entraînements

. données de verbalisation lors

d'entretiens en auto-

confrontation, menés 2 à 3

heures après l'entraînement

- Le traitement des données a été

opéré à deux niveaux :

. un codage et une catégorisation

de l'action des entraîneurs, à

partir de la méthode de la théorie ancrée (Strauss \& Corbin, 1990)

. classification des actions en

catégories plus larges appelées

" séries » (Theureau, 1992)

- D’Arripe-Longueville, Saury ; - Phases de coaching durant deux Fournier \& Durand (2001)

- Tir à l’arc

- But : étudier l'organisation temporelle et contextuelle des interactions entre l'entraîneur

compétitions

- Durée : 8 heures pour la

première et 2 jours pour la

seconde

- Deux types de données ont été retenues : les athlètes en compétition.

équipe de France, expérimentés dans le sport de haut niveau

depuis plus de 10 ans.

- ces entraîneurs ont été choisis à partir des critères de Côté et al

(1995).

- les joueurs étaient seniors féminins, juniors féminins et

masculins et appartenaient aux équipes nationales.

. des données d'observation et

d'enregistrement durant la

compétition

. des entretiens en auto-

confrontation à partir de 15

situations répondant à 4 critères

- traitement des données selon

une partie de la méthodologie de

l'analyse sémio-logique du cours d'action.

- Sève, Saury, Theureau \&

- 4 matches qui représentent un

Durand (2002)

- Tennis de table

enjeu compétitif important

tournois qualificatifs

- Buts : décrire l'apprentissage

au cours de la compétition et pour les Jeux Olympiques de Sydney l'équipe de France senior

(participation aux Jeux
- 1 entraîneur expert (critères de

Côté et al, 1995a)

Les conditions de respect
l'autonomie des athlètes

Cóminines de l'équipe

de France (2 faisaient partie des

8 meilleurs mondiaux, 2 avaient

des titres nationaux

\section{- 3 pongistes membres de}

Olympiques d'Atlanta et

champions d'Europe et vice

perçues des situations par

l'entraîneur :

la volonté de l'athlète

défavorable, l'entraîneur

propres résultats.

les adapter à leur propre fonctionnement. l'action (organisation, définition, d'optimalisation de l'efficacité guidage, soutien, surveillance,

bilan de l'action des joueurs)

- la dynamique temporelle de

l'action au cours de la séance.

Elle s'est basée sur la

progression et la répétition de

l'action des entraîneurs.

L'action des entraîneurs s'est

inscrite dans une temporalité.

Celle-ci était contrainte par les

compétitions importantes.

épendaient des caractéristiques

en situation perçue comme

favorable l'entraîneur respectait

. en situation perçue comme

encourageait l'athlète à initier

l'interaction et à interpréter ses

Les archers ont redéfinit les

instructions de l'entraîneur pour

Les joueurs faisaient preuve de

deux modes d'engagement dans

la situation : tester l'efficacité

des certaines actions face à

l'adversaire (exploration) et de leur intervention.

'action des entraîneurs a pris en compte deux temporalités

distinctes liées aux échéances

compétitives et à l'évolution du

joueur. Elle a induit une

structuration du temps et du type

de travail proposé au joueur.

Cette étude se situait dans une

perspective de compréhension de

l'action des entraîneurs

(caractérisation des situations

d'entraînement, rôle des

cognitions planificatrices,

modalités de développement de

l'expertise des entraîneurs).

Les résultats ont mis en évidence une conception particulière de

'aide à la performance au cours

de la compétition, qui s'est

fondée sur le respect de

l'autonomie et le développement

de la responsabilité de l'athlète

L'intervention de l'entraîneur a

émergé en situation critique ou

en situation de pression

temporelle élevée. Elle était

plutôt exploratoire.

Le coaching est apparu comme la conséquence d'une construction

locale et collective (entraîneur / athlète).

L’activité des pongistes en

situation compétitive contenai

une composante d'exploration et

d'apprentissage.

L'apprentissage était différent de 


\begin{tabular}{|c|c|c|c|c|}
\hline $\begin{array}{l}\text { identifier des processus utilisés } \\
\text { comme repères pour l'analyse } \\
\text { des processus mobilisés dans la } \\
\text { vie quotidienne. }\end{array}$ & $\begin{array}{l}\text { - Durée des matches : entre } 25 \text { et } \\
48 \text { min } \\
\text { - } 2 \text { types de recueil de données : } \\
\text {. données d'enregistrement au } \\
\text { cours de la compétition } \\
\text {. données de verbalisation au } \\
\text { cours d'entretiens en auto- } \\
\text { confrontation, d'une durée } \\
\text { moyenne de } 130 \text { min et menés } \\
\text { entre deux et six jours après les } \\
\text { matches. } \\
\text { - traitement des données selon le } \\
\text { cadre de l'analyse sémio-logique } \\
\text { du cours d'action. }\end{array}$ & $\begin{array}{l}\text { champion du monde par équipes } \\
\text { au moment de l'étude) }\end{array}$ & $\begin{array}{l}\text { marquer (exécution). } \\
\text { On a observé le passage d'un } \\
\text { engagement à la fois exploratoire } \\
\text { et exécutoire en début de match à } \\
\text { un engagement à dominante } \\
\text { exécutoire en fin de match. } \\
\text { Les pongistes construisaient et } \\
\text { utilisaient des connaissances au } \\
\text { cours du match. Cette } \\
\text { construction s'opérait soit sur la } \\
\text { base d'une ou de plusieurs } \\
\text { observations, soit en mettant en } \\
\text { relation des connaissances déjà } \\
\text { construites. }\end{array}$ & $\begin{array}{l}\text { celui observé au cours de } \\
\text { l'entraînement. } \\
\text { Il a semblé que l'entraînement se } \\
\text { centrait surtout sur le } \\
\text { développement d'habiletés } \\
\text { motrices et non sur des } \\
\text { compétences exploratoires } \\
\text { La construction des } \\
\text { connaissances était située (dans } \\
\text { et pour le match). }\end{array}$ \\
\hline $\begin{array}{l}\text { - Hauw, Berthelot \& Durand } \\
\text { (2003) } \\
\text { - Trampoline } \\
\text { - But : analyser l'expertise à } \\
\text { partir de modifications du } \\
\text { couplage situation - action, } \\
\text { montrer le caractère variable de } \\
\text { la performance experte }\end{array}$ & $\begin{array}{l}\text {-52 enchaînements en } \\
\text { compétition (de } 1999 \text { à 2002) } \\
\text { enchaînement de } 10 \text { actions } \\
\text {-3 types de recueil de données : } \\
\text {. données d'enregistrement au } \\
\text { cours de la compétition } \\
\text {. données de verbalisation au } \\
\text { cours d'entretiens en auto- } \\
\text { confrontation } \\
\text {. notes et observations au cours } \\
\text { de la compétition } \\
\text { - traitement des données selon le } \\
\text { cadre de l'analyse sémio-logique } \\
\text { du cours d'action. }\end{array}$ & $\begin{array}{l}\text { - } 10 \text { athlètes de l'équipe de } \\
\text { France ( } 8 \text { hommes et } 2 \text { femmes) } \\
\text {. } 3 \text { faisaient partie des } 5 \text { meilleurs } \\
\text { mondiaux, } 5 \text { des } 20 \text { meilleurs } \\
\text { mondiaux, } 2 \text { juniors titrés au plan } \\
\text { européen }\end{array}$ & $\begin{array}{l}\text { L'action était couplée à la } \\
\text { situation. La performance était } \\
\text { plutôt liée au contexte qu'à un } \\
\text { état stabilisé. Il existait plusieurs } \\
\text { modèles d'organisation de la } \\
\text { performance : } \\
\text { - structuration forte des phases } \\
\text { prises en compte dans la } \\
\text { modification de l'organisation } \\
\text { des actions, dans l'enchaînement } \\
\text { - plus grande liberté pour } \\
\text { modifier l'organisation des } \\
\text { actions au cours de } \\
\text { l'enchaînement, } \\
\text { - meilleure stabilité dans les } \\
\text { structures des cours d'action en } \\
\text { cas de difficulté. }\end{array}$ & $\begin{array}{l}\text { L’expertise des trampolinistes } \\
\text { était homogène. } \\
\text { Leur expertise n'était pas stable : } \\
\text { il existait une marge de } \\
\text { variabilité dans l'organisation du } \\
\text { cours d'action en fonction du } \\
\text { niveau. } \\
\text { L'action en contexte présentait } \\
\text { un caractère d'unicité. } \\
\text { L’expertise était située : elle était } \\
\text { définie par les couplages } \\
\text { successifs entre action et } \\
\text { situation. } \\
\text { Le mode d'organisation de } \\
\text { l'action dépendait du niveau } \\
\text { d'expertise. }\end{array}$ \\
\hline
\end{tabular}


Tableau 5

L'Etude liée à l'Activité de l'Expert en Référence au Modèle de la Suffisance cognitive

\begin{tabular}{|c|c|c|c|c|}
\hline Auteurs et études & Protocoles & Sujets (participants) & Résultats & Synthèse \\
\hline $\begin{array}{l}\text { - Macquet (2001) } \\
\text { - Volley-ball } \\
\text { - Buts : comprendre les } \\
\text { fondements de la compréhension } \\
\text { et de la décision des joueurs, le } \\
\text { degré de contrôle auquel elle } \\
\text { contribuait. }\end{array}$ & $\begin{array}{l}\text { - } 1 \text { match de } 1 / 4 \text { de Finale de } \\
\text { Championnat de France, } \\
\text { qualificatif pour les } \\
\text { championnats d’Europe } \\
\text { - durée du match : } 90 \text { minutes } \\
\text { - } 2 \text { types de recueil des données : } \\
\text {. données d'enregistrement de la } \\
\text { totalité du match (une bande } \\
\text { pour l'ensemble du match et une } \\
\text { bande par joueur en plan large) } \\
\text {. données de verbalisation issues } \\
\text { de l'entretien en auto- } \\
\text { confrontation (7 entretiens de } 90 \\
\text { minutes avec chacun des joueurs, } \\
\text { confrontés à deux écrans) } \\
\text { - traitement des données : } \\
\text { découpage des situations de jeu, } \\
\text { puis des verbalisations en } \\
\text { fonction de catégories inductives, } \\
\text { basées sur la théorie ancrée. }\end{array}$ & $\begin{array}{l}\text { - } 7 \text { joueurs d'une équipe de } \\
\text { Nationale } 1 \mathrm{~A} \\
\text { - } 10 \text { ans d'expérience en volley- } \\
\text { ball (sauf pour un joueur : } 6 \text { ans) }\end{array}$ & $\begin{array}{l}5 \text { niveaux de situation ont été } \\
\text { mis en évidence en fonction du } \\
\text { niveau de compréhension de la } \\
\text { situation par le joueur. } \\
\text { Il existait une relation entre le } \\
\text { niveau de compréhension des } \\
\text { situation et le niveau de contrôle } \\
\text { de processus. } \\
\text { La compréhension s'appuyait à } \\
\text { la fois sur la perception } \\
\text { d'informations disponibles (ou } \\
\text { sur l'anticipation d'informations) } \\
\text { et sur l'utilisation ou sur la } \\
\text { construction de connaissances en } \\
\text { cours d'action. } \\
\text { La variété des informations et } \\
\text { des connaissances rapportées } \\
\text { était proportionnelle à la pression } \\
\text { temporelle perçue. }\end{array}$ & $\begin{array}{l}\text { Le joueur ne pouvait pas tout } \\
\text { comprendre : il devait se } \\
\text { satisfaire d'une compréhension } \\
\text { suffisante pour agir. } \\
\text { Cette compréhension présentait } \\
\text { un coût cognitif important (il ne } \\
\text { s'y est pas engagé } \\
\text { systématiquement). } \\
\text { Les décisions étaient planifiées } \\
\text { avec des adaptations en ligne (ou } \\
\text { émergentes). } \\
\text { Les performances des experts } \\
\text { n’était pas toujours } \\
\text { exceptionnelles : ils tentaient de } \\
\text { maîtriser certaines situations au } \\
\text { détriment d’autres, qui } \\
\text { apparaissaient plus difficiles. }\end{array}$ \\
\hline
\end{tabular}

